Draft Version November 5, 2018

Preprint typeset using $\mathrm{L}^{A} \mathrm{~T}_{\mathrm{E}} \mathrm{X}$ style emulateapj v. 08/22/09

\title{
THE TEMPERATURE OF INTERSTELLAR CLOUDS FROM TURBULENT HEATING
}

\author{
LiUBin PAN AND PAOLO PADOAN \\ Department of Physics, University of California, San Diego, CASS/UCSD 0424, 9500 Gilman Drive, La Jolla, CA 92093-0424; \\ lpan@ucsd.edu, ppadoan@ucsd.edu \\ Draft version November 5, 2018
}

\begin{abstract}
To evaluate the effect of turbulent heating in the thermal balance of interstellar clouds, we develop an extension of the log-Poisson intermittency model to supersonic turbulence. The model depends on a parameter, $d$, interpreted as the dimension of the most dissipative structures. By comparing the model with the probability distribution of the turbulent dissipation rate in a simulation of supersonic and super-Alfvénic turbulence, we find a best-fit value of $d=1.64$. We apply this intermittency model to the computation of the mass-weighted probability distribution of the gas temperature of molecular clouds, high-mass star-forming cores, and cold diffuse HI clouds. Our main results are: i) The mean gas temperature in molecular clouds can be explained as the effect of turbulent heating alone, while cosmic ray heating may dominate only in regions where the turbulent heating is low; ii) The mean gas temperature in high-mass star-forming cores with typical FWHM of $\sim 6 \mathrm{~km} \mathrm{~s}^{-1}$ (corresponding to a 1D rms velocity of $2.5 \mathrm{~km} \mathrm{~s}^{-1}$ ) may be completely controlled by turbulent heating, which predicts a mean value of approximately $36 \mathrm{~K}$, two to three times larger than the mean gas temperature in the absence of turbulent heating; iii) The intermittency of the turbulent heating can generate enough hot regions in cold diffuse $\mathrm{HI}$ clouds to explain the observed $\mathrm{CH}^{+}$abundance, if the rms velocity on a scale of $1 \mathrm{pc}$ is at least $3 \mathrm{~km} \mathrm{~s}^{-1}$, in agreement with previous results based on incompressible turbulence. Because of its importance in the thermal balance of molecular clouds and high-mass star-forming cores, the process of turbulent heating may be central in setting the characteristic stellar mass and in regulating molecular chemical reactions.
\end{abstract}

Subject headings: ISM: kinematic and dynamics — turbulence

\section{INTRODUCTION}

The dissipation of turbulent kinetic energy provides a potentially important heating source in a variety of Galactic astrophysical environments, such as the solar wind (e.g., Matthaeus et al. 1999), interstellar clouds (e.g., Falgarone and Puget 1995), and the warm ionized medium (e.g., Minter and Balser 1997). The effect of turbulent heating has also been studied in extragalactic environments, such as in the context of the broad-line regions of quasars (Bottorff \& Ferland 2002), and in intracluster cooling flows (Dennis \& Chandran 2005).

Astrophysical turbulence is often highly supersonic and magnetized. Energy decay in supersonic turbulence was thought to be very rapid due to shocks (e.g., Goldreich and Kwan 1974), while the presence of strong magnetic fields was believed to suppress the compressible modes and thus reduce the dissipation rate (Arons \& Max 1975). Recent numerical simulations offered a better understanding of energy dissipation in supersonic MHD turbulence (Stone et al. 1998, Mac Low et al. 1998, Padoan and Norlund 1999). As in incompressible hydrodynamic turbulence, where the kinetic energy decays in a turnover time of the largest eddies, the dissipation timescale in supersonic MHD turbulence is of order the flow crossing timescale (Stone et al. 1998) or, equivalently, the dynamical timescale at the driving scale (Mac Low 1999).

In typical molecular clouds the dynamical timescale is rather short, of order a million year, suggesting the need of continuous energy injection to support the observed turbulence. This result also implies a large turbulent heating rate. An estimate of the average dissipation rate from this timescale shows that it can be several times larger than the cosmic-ray heating rate, and thus may be the primary heating source in molecular clouds. Stone et al. (1998) also argued that the average turbulent heating rate can be comparable to the photoelectric heating in HI clouds with large velocity dispersions (however, according to Wolfire et al. 2003, the overall turbulent heating in the neutral medium may not be sufficient to produce the observed CII luminosity). In this paper, we provide a general theoretical formulation of the problem of turbulent heating, and investigate its effect on various types of interstellar clouds.

Turbulent dissipation is characterized by its strong spatial roughness (see Figures 11 and 21). Extreme dissipation events appear in the smallest structures occupying a tiny volume or mass fraction, while a significant fraction of the flow experiences essentially no dissipation. This implies a broad probability distribution of the dissipation rate, which must be taken into account for a consistent investigation of turbulent heating. The extended tail of this distribution at large dissipation rates, corresponding to highly dissipative structures, is responsible for the anomalous scaling of the high-order velocity structure functions, referred to as intermittency in turbulence theory (Frisch 1995).

Intermittency has been extensively studied in incompressible turbulence. The intermittent model by She and Leveque (1994), which considers a hierarchy of dissipation rates of different levels and relates them to the fractal dimension of the most intermittent dissipative structures, has been very successful in reproducing the mea- 
sured scaling exponents of structure functions in incompressible turbulence. It has been shown that the model is equivalent to a log-Poisson distribution of the dissipation rate (Dubrulle 1994, She and Waymire 1995). The probability distribution of the dissipation rate in supersonic turbulence has not been studied yet. Although the She and Leveque model with a fractal dimension of 2 , corresponding to shocks, agrees well with the structure functions computed from numerical simulations of highly compressible turbulence (Boldyrev et al. 2002, Padoan et al. 2004), it remains to be confirmed whether the distribution of the dissipation rate in supersonic turbulence is consistent with a log-Poisson process. This theoretical concern and the wide application of an intermittency model of turbulent heating to various astrophysical environments are the primary motivations of the present work.

An important effect of the intermittency of the turbulent dissipation is the generation of small regions with very large heating rate, and thus very high temperature. Falgarone and Puget (1995) were probably the first to recognize the importance of this effect in cold HI clouds. Observed molecules, such as $\mathrm{CH}^{+}$, suggest the existence of hot regions in cold HI clouds, because their production requires temperatures much higher than the average. Adopting experimental results from incompressible turbulent flows, they found that strong local turbulent heating in HI clouds could produce a sufficient fraction of hot regions to explain the observed abundance of $\mathrm{CH}^{+}$ molecules. In this work we address the validity of their result in the case of an intermittency model for supersonic turbulence, more appropriate for cold HI clouds.

In $\S 2$, we study the intermittent energy dissipation in supersonic turbulence. We show that the log-Poisson intermittency model gives probability distributions for the dissipation rate in excellent agreement with those at the resolved scales of numerical simulations of supersonic turbulence. In $\S 3$, we discuss heating and cooling processes in the interstellar medium and give the energy balance equations. We apply the log-Poisson intermittency model to investigate turbulent heating in molecular clouds, high-mass star-forming cores, and cold HI clouds in $\S 4$. Discussions and conclusions are given in $\S 5$.

\section{INTERMITTENCY OF TURBULENT HEATING}

The energy dissipation rate in turbulent flows is known to exhibit fluctuations in both space and time. The viscous dissipation rate per unit mass is given by

$$
\epsilon(\mathbf{x}, t)=\nu\left((\nabla \times \mathbf{v})^{2}+\frac{4}{3}(\nabla \cdot \mathbf{v})^{2}\right)
$$

where $\nu$ is the kinematic viscosity, and we have omitted the bulk viscosity, which is negligible for an ideal gas (a good approximation for the interstellar medium). The divergence term in eq. (11) is necessary for compressible flows. Clearly, in the presence of a fluctuating velocity field, the dissipation rate is expected to be inhomogeneous. Kolmogorov's 1941 theory uses the average dissipation rate (as the energy transfer rate in the inertial range) and thus implicitly assumes spatial homogeneity in the dissipation rate. That theory is therefore not sufficient to study turbulent heating. Because a significant fraction of the kinetic energy is viscously dissipated in the finest structures, such as vortex tubes and shocks (in supersonic turbulence), which occupy only a small volume fraction (or mass fraction for compressible turbulence), the dissipation rate is strongly intermittent. Its probability distribution has a tail much more extended than that of a Gaussian distribution, as the result of strong local dissipative events. A careful consideration of this intermittent distribution is essential for investigating turbulent heating. In the following, we briefly review the intermittency theory.

\subsection{Intermittency Theory}

Kolmogorov (1962) and Oboukhov (1962) developed the first intermittency model for incompressible turbulence. To account for the fluctuations in the dissipation rate, they defined the dissipation rate, $\epsilon_{l}$, at each scale $l$ and considered its probability distribution as a function of $l$, which, together with the refined self-similarity hypothesis (Kolmogorov 1962), was used to predict the scaling exponents of the velocity structure functions. Generalizing to a compressible flow, the dissipation rate per unit mass at a scale $l$ is,

$$
\epsilon_{l}(\mathbf{x}, t)=\frac{1}{\rho_{l}(\mathbf{x}, t) V(l)} \int_{\left|\mathbf{x}^{\prime}\right|<l} \rho\left(\mathbf{x}+\mathbf{x}^{\prime}, t\right) \epsilon\left(\mathbf{x}+\mathbf{x}^{\prime}, t\right) d \mathbf{x}^{\prime}
$$

where $V(l)=4 \pi l^{3} / 3$ is the volume of a spherical region of size $l$ and

$$
\rho_{l}(\mathbf{x}, t)=\frac{1}{V(l)} \int_{\left|\mathbf{x}^{\prime}\right|<l} \rho\left(\mathbf{x}+\mathbf{x}^{\prime}, t\right) d \mathbf{x}^{\prime}
$$

is the average density of that spherical region. From its definition, $\epsilon_{l}$ is a function of $\mathbf{x}$ and we aim to study its spatial fluctuations. Note that the average rate, $\epsilon_{l}$, over a region of size $l$ defined here is different from $\nu v_{l}^{2} / l^{2}$ used by Falgarone and Puget (1995), which neglects the velocity gradient fluctuations in the region, and thus is valid only for $l$ smaller than the Kolmogorov dissipation scale, $\eta$, below which the velocity field is smooth and the velocity gradient is approximately constant.

We point out that the pdf needed to study turbulent heating is that at the dissipation scale, the smallest scale of fluctuations in the dissipation rate. For this purpose, an ideal intermittency model to use is one for the dissipation range. However, most intermittency models, including the log-Poisson model we will adopt below, are for the inertial scales, and we are not aware of theoretical models that give the pdf of the dissipation rate in the dissipation range. Here, we take the following approach to obtain the pdf at the dissipation scale. We first focus on the inertial range and test an intermittency theory for the dissipation rate pdf in the inertial scales by comparing its predicted pdf at each scale with that computed from numerical simulations. If the theoretical pdf and its scale dependence agree with those from the simulation data, we extrapolate the model to obtain the pdf at the dissipation scale. This assumes that the pdf at the dissipation scale can be approximated by that extrapolated from the inertial scales. Although there is no theoretical estimate for its accuracy, the assumption is justified by the expectation that the pdf in the dissipation range probably connects continuously and smoothly 
to that in the inertial range and is supported by our numerical results (see below).

The probability distribution, $P\left(\epsilon_{l}\right)$, of the dissipation rate, $\epsilon_{l}$, at a scale $l$ can be calculated as

$$
P\left(\epsilon_{l}\right)=\frac{1}{\bar{\rho} V} \int \delta\left(\epsilon_{l}-\epsilon_{l}(\mathbf{x}, t)\right) \rho_{l}(\mathbf{x}, t) d \mathbf{x}
$$

where $V$ is the total volume of the system and $\bar{\rho}$ is the overall average density in the flow. We have used the density as a weighting factor $\left(\rho_{l} / \bar{\rho}\right.$, which is unity for incompressible turbulence) to account for the density variations in supersonic turbulence. This density or mass weighting is supported by Kritsuk et al. (2007), who find that replacing the velocity field $\mathbf{v}$ by $\rho^{1 / 3} \mathbf{v}$ gives a 3rd order structure function in supersonic turbulence in agreement with Kolmogorov's 4/5 law. This is equivalent to saying that the density-weighted average dissipation rate, $\left\langle\epsilon_{l}\right\rangle$, is the same over all the inertial scales. This is built in our definition, eq. (4),

$$
\left\langle\epsilon_{l}\right\rangle=\int \epsilon_{l} P\left(\epsilon_{l}\right) d \epsilon_{l} \propto \int \rho_{l}(\mathbf{x}, t) \epsilon_{l}(\mathbf{x}, t) d \mathbf{x}
$$

which is independent of $l$, as follows from eq. (2). We will therefore denote the average dissipation rate $\left\langle\epsilon_{l}\right\rangle$ as $\bar{\epsilon}$. Clearly, $\bar{\epsilon} \simeq U^{3} / L$, where $U$ is the rms velocity of the flow and $L$ is the integral scale. Another reason for choosing the density weighted distribution is that we want to estimate the mass-weighted average temperature resulting from the turbulent heating of interstellar clouds.

Kolmogorov (1962) and Oboukhov (1962) proposed a log-normal distribution for $\epsilon_{l}$, for which Yaglom (1966) gave a justification with a self-similar eddyfragmentation argument. The variance of the log-normal distribution is assumed to be proportional to the number of cascade steps, $\propto \ln (L / l)$, from the integral scale, $L$, to the scale of interest, $l$. Experiments by Ansemet et al. (1984) find that the scaling exponents of the structure functions agree with the log-normal model at low orders, but depart from it at orders $\gtrsim 10$. The lognormal model has also been shown to violate some theoretical requirements on the structure function exponents in incompressible turbulence (Frisch 1995). Numerical simulations find that the log-normal distribution of the dissipation rate is quite a good approximation in the inertial range and is valid up to about 4-5 $\sigma$, beyond which a departure from the simulations is clearly seen (e.g., Yueng et al. 2006). This departure explains the deviation of the model from the measured structure functions in the experiments. In this work we do not consider the log-normal model (which was proposed for incompressible turbulence), because it gives a poor fit to $P\left(\epsilon_{l}\right)$ computed in our simulations of supersonic turbulence.

A different intermittency model has recently been proposed by She and Leveque (1994). The scaling exponents of structure functions of order up to 10 predicted by this model for incompressible hydrodynamic turbulence agree with experimental data with an accuracy of $1 \%$. In their original paper, She and Leveque start from the hierarchy of dissipation rates at each scale. By invoking a "hidden symmetry" that relates the dissipation rate at different intensity levels to the strongest dissipative structures at a given scale $l$, they determine the whole hierarchy of dissipation rates from the fractal dimension of the most intermittent structures and from the scaling of the dissipation rate of these structures with $l$. The "hidden symmetry" was speculated to be an unknown symmetry of the Navier-Stokes equation and was immediately recognized as corresponding to a log-Poisson process in a multiplicative energy cascade model.

The model has been successfully applied to incompressible MHD turbulence (Muller and Biskamp 2000), supersonic turbulence (Boldyrev et al. 2002) and superAlvénic turbulent flows (Padoan et al. 2004). These studies compute the scaling exponents of the structure functions from numerical simulations and compare them to the prediction of the log-Poisson model with different fractal dimensions for the most intermittent structures. They find that a 2D geometry of the most intermittent structures, corresponding to current sheets in MHD turbulence or shocks in highly supersonic turbulence, gives an excellent fit to the simulation results. Here, instead of considering the scaling exponents of the structure functions, we focus on the log-Poisson version of the SheLeveque model and compare the model directly with the probability distribution, $P\left(\epsilon_{l}\right)$, measured in simulations of supersonic MHD turbulence. Our purpose is to check whether this model agrees with numerical simulations. If so, we can first "calibrate" it by fitting resolved inertial scales in the simulations, and then extrapolate it to high Reynolds number flows. In the following, we give a brief description of the log-Poisson model. The interested reader is referred to Dubrulle (1994) and to She and Waymire (1995) for more details (see also Pan et al. 2008, which includes both the original presentation by She and Leveque and the log-Poisson version).

\subsection{The Log-Poisson Model}

The log-Poisson model is a multiplicative cascade model where the dissipation rates at two scales, $l_{1}$ and $l_{2}$, are related by a multiplicative factor, $W_{l_{1} l_{2}}$,

$$
\epsilon_{l_{2}}=W_{l_{2} l_{1}} \epsilon_{l_{1}}
$$

Since the average dissipation rate at each scale is equal to the overall average dissipation rate $\bar{\epsilon}$ (see above), the average of the multiplicative factor, $\left\langle W_{l_{2} l_{1}}\right\rangle$, is required to be unity. According to the speculation by She and Waymire (1995), two types of events determine $W_{l_{1} l_{2}}$. One is the amplification of the dissipation rate that tends to cause singular structures at small scales. The other, called the modulation-defects by She and Waymire, corresponds to the failure of structures at scale $l_{1}$ to turn into the most dissipative structures at scale $l_{2}$ in the cascade. The first type of events is assumed to give an amplification factor of $\left(l_{1} / l_{2}\right)^{\gamma}$, which approaches infinity as $l_{2} \rightarrow 0$ for positive $\gamma$. The parameter $\gamma$ measures how intense the most intermittent structures at scale $l_{2}$ can be. Assuming that the kinetic energy per unit mass available for dissipation in the most intermittent structures is $\sim U^{2}$, where $U$ is the rms velocity at the integral scale, and that the dynamical timescale $t_{l}$ in these structures follows the regular Kolmogorov scaling, $t_{l} \propto l^{2 / 3}$, She and Leveque (1994) argued that the energy dissipation rate in these intense structures is $\sim U^{2} / t_{l} \propto l^{-2 / 3}$, which suggests that $\gamma=2 / 3$. The modulation-defects are assumed to be a discrete process, where each defect reduces $W_{l_{1} l_{2}}$ by a factor of $\beta$ and the number of defects, 


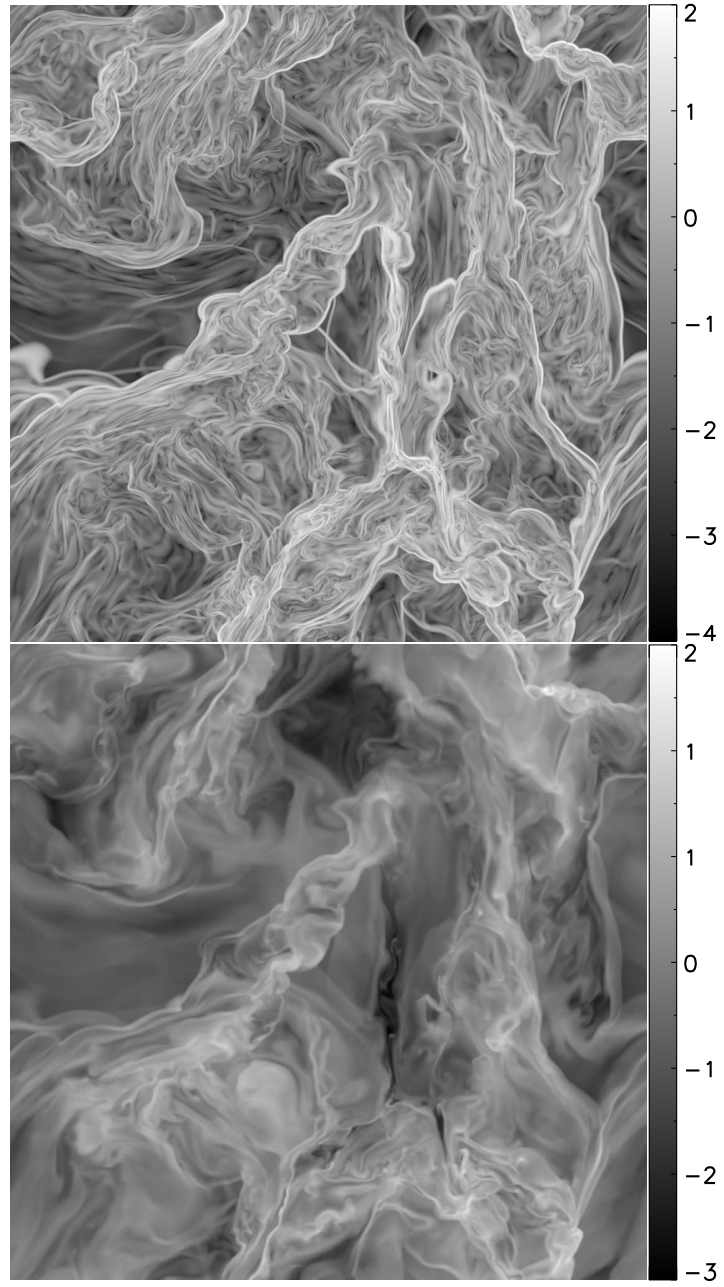

FIG. 1.- Logarithm of the dissipation rate $(\log (\epsilon(\mathbf{x}) / \bar{\epsilon})$, upper panel) and density $(\log (\rho(\mathbf{x}) / \bar{\rho})$, lower panel) relative to their averages on a slice of a snapshot of the simulation. Both the dissipation rate and the density have a filamentary structure. Dense filaments are usually sites of strong dissipation, but filaments of high dissipation rate are also found at the interface of low and high density regions. As a result, the dissipation rate and the gas density are practically uncorrelated (see Fig. 3).

$n$, occurring in the cascade from $l_{1}$ to $l_{2}$ is assumed to obey a Poisson distribution. The physical meaning of the parameter $\beta$ will be discussed below. These arguments are represented by two equations,

$$
W_{l_{2} l_{1}}=\left(l_{1} / l_{2}\right)^{\gamma} \beta^{n}
$$

and

$$
P(n)=\exp \left(-\lambda_{l_{1} l_{2}}\right) \frac{\lambda_{l_{1} l_{2}}^{n}}{n !}
$$

where $\lambda_{l_{1} l_{2}}$ is the average number of defects in the cascade from $l_{2}$ to $l_{1}$. Requiring $\left\langle W_{l_{1} l_{2}}\right\rangle=1$ gives $\lambda_{l_{1} l_{2}}=$ $\gamma \ln \left(l_{1} / l_{2}\right) /(1-\beta)$. As expected, $\lambda_{l_{1} l_{2}}$ is proportional to the number of steps in the cascade, $\ln \left(l_{1} / l_{2}\right)$.

Taking the logarithm of eqs. (6) and (7), we see that the distribution of $\epsilon_{l}$ is $\log$-Poisson. Given the probability distribution at one scale, the distribution of $\epsilon_{l}$ at any other scale $l$ can be derived using eqs. (6), (7) and (8). The convenient scale to start with is the integral scale, $L$, where the distribution, $P_{L}$, of the dissipation rate mainly depends on the forcing of the flow and thus is probably flow-dependent. However, the dissipation rate, $\epsilon_{L}$, at $L$

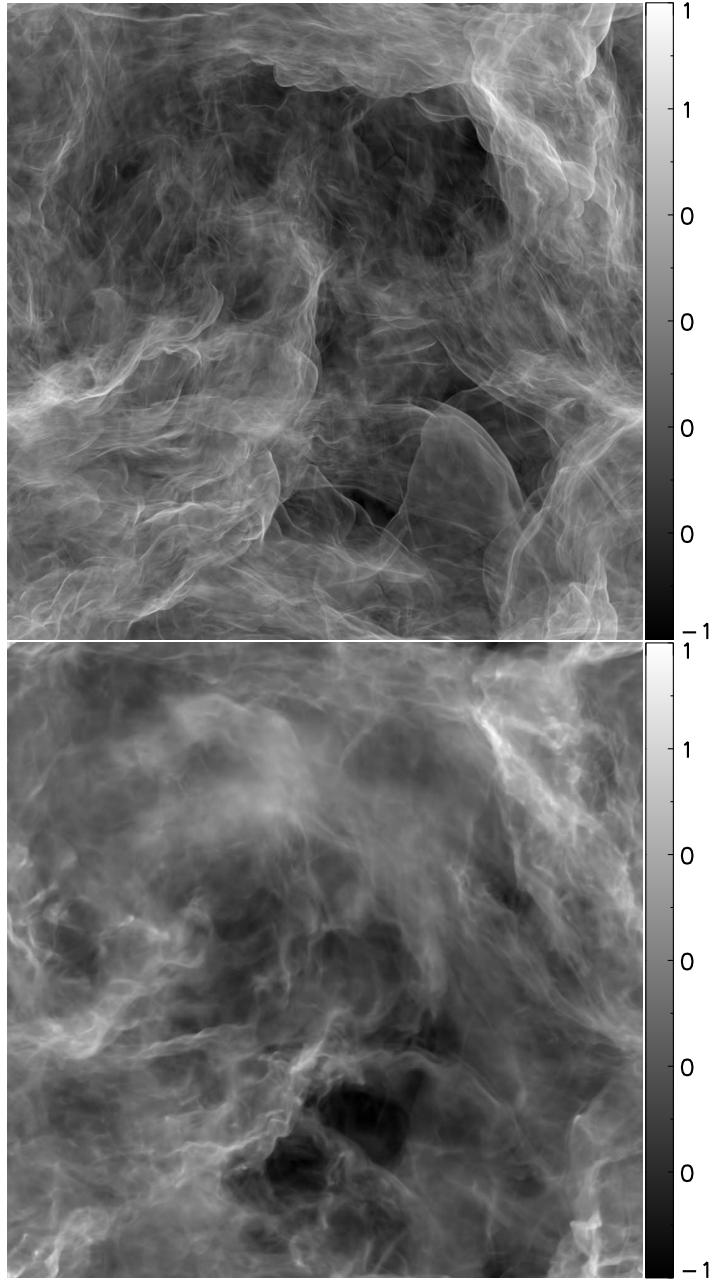

FIG. 2.- Logarithm of the projected dissipation rate (upper panel) and of the projected density (lower panel) relative to their averages, from a snapshot of the simulation. As a result of the lack of correlation between the two quantities (see Fig. 3), individual structures in the projected dissipation rate do not have an obvious counterpart in the projected density.

is close to the average, $\bar{\epsilon}$, and hence $P_{L}$ is expected to be narrow and approximately a delta function. Given $P_{L}\left(\ln \epsilon_{L}\right)$, we find that

$P\left(\epsilon_{l}\right) d \epsilon_{l}=$

$\sum_{n=0}^{\infty} \exp (-\lambda) \frac{\lambda^{n}}{n !} P_{L}\left(\ln \left(\epsilon_{l} / \bar{\epsilon}\right)-\gamma \ln (L / l)-n \ln (\beta)\right) d \ln \left(\epsilon_{l} / \bar{\epsilon}\right)$

where $\lambda=\lambda_{L l}=\gamma \ln (L / l) /(1-\beta)$. Note that if $P_{L}$ is a delta function, then $P\left(\epsilon_{l}\right)$ is discrete with a series of spikes.

The physical meaning of the parameter $\beta$ in the logPoisson version of the model is not clear. Deriving the moments of the distribution $P\left(\epsilon_{l}\right)$ from eq. (9) and comparing with the original version of the model, one finds that $\beta$ here corresponds to the same $\beta$ parameter introduced in the "hidden symmetry" by She and Leveque (1994), which has been related to the fractal dimension of the most intermittent structures, $d$, and the parameter $\gamma$ by $\gamma /(1-\beta)=D-d$, with $D=3$ being the dimension of the system. Here we omit the derivation of this relation that can be found, e.g., in Pan et al. (2008). For vortex tubes, the dimension is $d=1$ and for shocks 
$d=2$. Because in supersonic turbulence about $1 / 3$ of the energy is dissipated in dilatational modes and $2 / 3$ in solenoidal modes (Kritsuk et al. 2007), $d$ is expected to be between 1 and 2 .

\subsection{The Log-Poisson Model for Supersonic Turbulence}

We compare the log-Poisson distribution, eq. (9), with results from a numerical simulation of supersonic MHD turbulence. We examine how well this model can be extended to supersonic MHD flows and what fractal dimension of the most intense dissipative structures gives the best fit to the simulation. We take 6 snapshots from the $1000^{3}$ Stagger-code MHD simulation in Padoan et al. (2007). The simulation adopts periodic boundary conditions, isothermal equation of state, random forcing in Fourier space at wavenumbers $1 \leq k \leq 2$ ( $k=1$ corresponds to the computational box size), uniform initial density and magnetic field, and random initial velocity field with power only at wavenumbers $1 \leq k \leq 2$. The simulation is both supersonic and super-Alfvénic, with an rms sonic Mach number of $\mathcal{M}_{\mathrm{s}} \approx 9$, and an initial rms Alfvénic Mach number of $\mathcal{M}_{\mathrm{a}, \mathrm{i}}=29.7$. After a few dynamical times, the magnetic energy is amplified by the turbulence, and the rms Alfvénic Mach number with respect to the rms magnetic field is $\mathcal{M}_{\mathrm{a}} \approx 2.8$, still super-Alfvénic.

In each snapshot, we evaluate the dissipation rate at the grid points according to eq. (11). A complication arises as a result of the limited numerical resolution. The dissipation rate should be calculated at the Kolmogorov scale using the kinematic viscosity, $\nu \simeq c /(n \sigma)$, where $c$ is the sound speed, $n$ the number density and $\sigma$ the collision cross section. This is not possible because the resolution scale in the simulation is much larger than the Kolmogorov scale. We cannot use the kinematic viscosity to calculate the dissipation rate at the grid points, because that neglects the fluctuations in velocity gradients below the grid scale and would underestimate the dissipation rate in each computational cell. To avoid this, we are forced to use an effective viscosity at the grid size to account for the sub-grid fluctuations erased by the numerical viscosity. In principle, the effective viscosity at each grid depends on the sub-grid fluctuations and (perhaps weakly) on the local Kolmogorov scale (which varies in space because the kinematic viscosity $\nu$ depends on density). Due to the lack of information about sub-grid scales, we simply assume the effective viscosity is constant, and normalize it so that the mean dissipation rate averaged over all the grid points is equal to the overall dissipation rate of the flow, $\bar{\epsilon} \simeq U^{3} / L$. With this assumption, we calculate the dissipation rate in each computational cell.

Figure 1 shows the logarithm of the dissipation rate (upper panel) and density (lower panel) on a slice of a snapshot of the simulation. Both the dissipation rate and the density have a filamentary structure. Dense filaments are usually sites of strong dissipation, but filaments of high dissipation rate are also found at the interface of low and high density regions. As a result, the dissipation rate and the gas density are practically uncorrelated. This lack of statistical correlation between dissipation rate and gas density is apparent when comparing images of projected dissipation rate and projected density, shown in Figure 2. Structures in these two im-

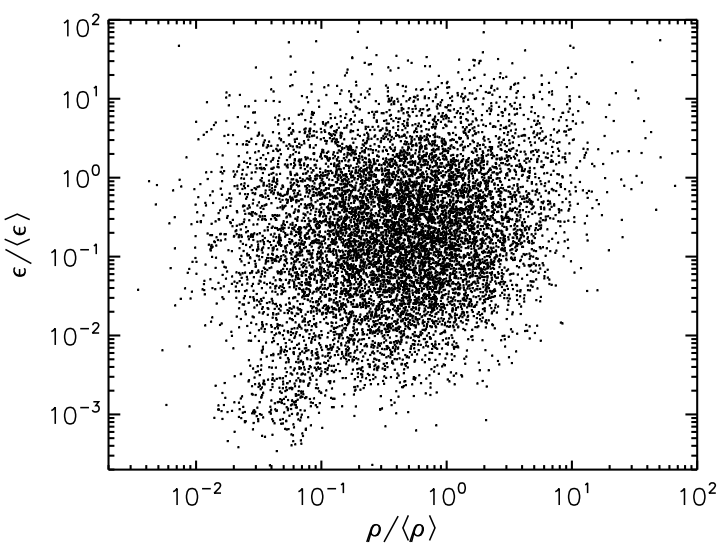

FIG. 3.- Dissipation rate versus gas density in 10,000 randomly sampled computational cells from one snapshot of the simulation. The dissipation rate and the gas density are practically uncorrelated. The correlation coefficient computed over all the computational cells of one snapshot is -0.11 .

ages appear to be rather independent of each other. The correlation coefficient between dissipation rate and gas density, computed over all the computational cells of one snapshot, is very low, -0.11 , as illustrated by the scatter plot of Figure 3. for 10,000 randomly selected computational cells of one snapshot.

From the dissipation rate at each computational cell, we compute the average dissipation rate, $\epsilon_{l}$, at any larger scale $l$, using eq. (2) (instead of calculating the average over spheres, we compute that over cubes of size $l$ from the simulation data). We then calculate the densityweighted probability distribution, $P\left(\epsilon_{l}\right)$, of the average dissipation rate, $\epsilon_{l}$, over regions of size $l$, using eq. (4). We carry out the same calculation for each snapshot and average the distributions from the 6 snapshots. The results are plotted in Figure 4 for $l=L / 64, L / 128, L / 256$, and $L / 512$, where $L$ is the size of the simulation box. The distribution becomes broader toward smaller scales, indicating the dissipation rate is more intermittent at smaller scales. Note that the distribution of $\log (\epsilon)$ is strongly skewed toward small values, suggesting that a log-normal model cannot give a satisfactory fit to the numerical results.

Figure 4 also shows the probability distribution from the log-Poisson model, eq. (9), with $\gamma=2 / 3$ and $\beta=0.51$ (which corresponds to a fractal dimension of $d=1.64$ for the most intense dissipative structures) at the same scales $l$. As mentioned above, at the box size $L, \epsilon_{L}=\bar{\epsilon}$ and $P_{L}$ is close to a delta function. We have set $P_{L}$ to be Gaussian with a small variance that is just large enough to make the distribution given by eq. (9) a smooth function. The log-Poisson model with a fractal dimension of the most dissipative structures $d=1.64$ gives an excellent agreement with numerical simulations over more than 5 orders of magnitudes in the dissipation rate. The agreement for $l=L / 128$ and $L / 256$ is especially remarkable, while slight departures for $l=L / 64$ and $L / 512$ are perhaps due to, respectively, the memory of large-scale motions and the suppression of intermittency by numerical dissipation. Note that the motivation of the logPoisson model was to explain the tail at large dissipation rates corresponding to high-order structure functions. It turns out that this model provides a surprisingly good 


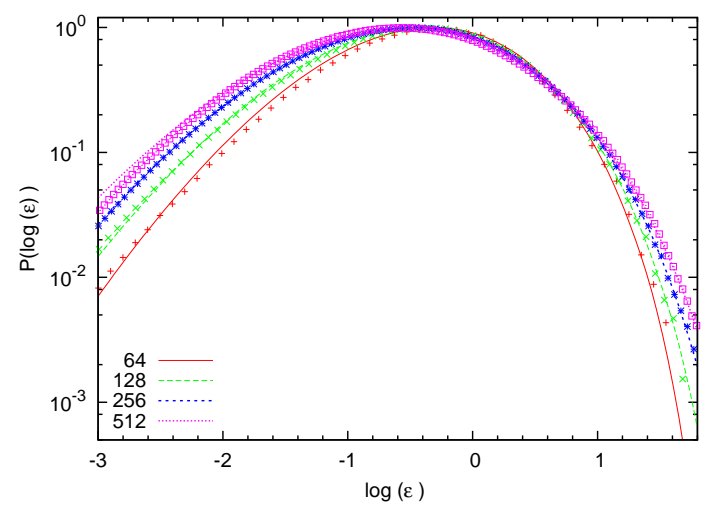

FIG. 4.- Density-weighted probability distribution of the dissipation rate at different scales. The symbols are from our simulation of supersonic MHD turbulence, and the curves from the log-Poisson model (eq. (9)) with $\gamma=2 / 3$ and $\beta=0.51$, corresponding to a fractal dimension $d=1.64$ of the most intermittent structures. The model gives an excellent fit to the distribution computed from the simulation. From bottom to top, the different symbols and the curves correspond to the distribution at $L / 64, L / 128, L / 256$, and $L / 512$, where $L$ is the computational box size. The distribution becomes broader toward smaller scales, indicating stronger intermittency at smaller scales.

fit to the tail of low dissipation rates as well. Padoan et al. (2004) obtained a fractal dimension of about 2 from MHD simulations with similar Mach numbers as here. In that paper, the structure functions were computed without density weighting, which may explain the difference in the resulting dimension of the dissipative structures. Kritsuk et al. (2007) found that two dimensions, $d=1.5$ and $d=2.25$, can give acceptable fit to the scaling exponents for the structure functions of $\rho^{1 / 3} \mathbf{v}$ in supersonic hydrodynamic turbulence. From their Fig. 2, the fitting quality is better for $d=1.5$, which is quite close to our result here. However, we point out that, except for the third order structure function (corresponding to the first order moment of the dissipation rate here), the densityweighting in $\rho^{1 / 3} \mathbf{v}$ is different from that in eq. (4).

To evaluate the uncertainty in the measured dimension $\mathrm{d}$ from the average pdf over 6 snapshots, we consider variations of the pdf from snapshot to snapshot, which supposedly include both the intrinsic temporal fluctuations of the pdf (if they exist) and the measurement uncertainty in each snapshot. We think the measurement uncertainty at small scales is probably small because of the large number of sampling cubes (thus we put more weight to the pdf towards smaller scales) and the snapshot-tosnapshot variations at these scales may primarily reflect the intrinsic temporal fluctuations (which corresponds to the variations in the predicted temperature from time to time). We calculated the standard deviation of $\log (\mathrm{P})$ in different $\epsilon$ bins over the 6 snapshots. In Fig. 5, we plot the 1- $\sigma$ error bars around the data points for the pdf at $l=1 / 256 L$, which are normalized by the log-Poisson pdf with $d=1.64$. Error bars of similar size are also obtained for other scales (the size of error bars for the scale, L/64, is about $60 \%$ larger than those in Fig. 5. This is because the measurement uncertainty is larger due to the smaller number of sampling cubes.). We see that the snapshot-to-snapshot variation is quite small except at the far right tail (the effect of which will be discussed later in $\S 4.3$ ). From the two curves representing the logPoisson model with $d=1.56$ and $d=1.71$ (normalized

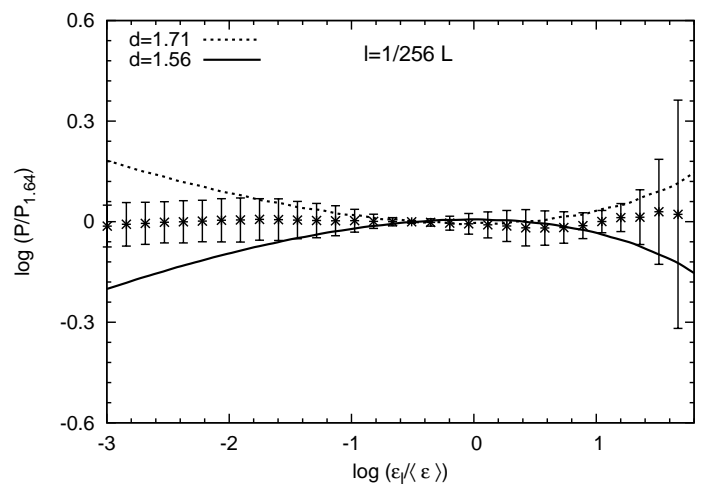

FIG. 5.- Snapshot-to-snapshot variations of the dissipation rate pdf at $l=L / 256$ in the simulation. The data points are the average pdf at $L / 256$ (the same asterisk data points in Fig. 4) normalized to the log-Poisson pdf eq. (9) with the best-fit dimension, $d=1.64$. The error bars indicate the standard deviation $(1-\sigma)$ of the pdf variations over the 6 snapshots. The error bars include both temporal variations of the pdf (if they exist) and measurement uncertainties. Also shown are the $\log$-Poisson pdfs with $d=1.56$ and $d=1.71$, also normalized to that with $d=1.64$. The predicted distribution from the model with $d$ in the range $(1.56-1.71)$ generally falls within the $1-\sigma$ error bars.

to that with $d=1.64$ ), we find that the model gives acceptable fits to the measured pdf within the 1- $\sigma$ error bars, for a range of values of the dimension $d$ between $d=1.56$ and $d=1.71$. We will evaluate the effect of this range of $d$ in our applications.

We have shown that the log-Poisson model agrees very well with the pdf from resolved scales in our simulations for supersonic MHD turbulence. As mentioned earlier, we are interested the dissipation rate pdf at the Kolmogorov scale, $\eta$, for applications to turbulent heating, and we assumed that the pdf at the scale $\eta$ can be obtained by extrapolating the log-Poisson model, proposed for the inertial range only, to the dissipation scale. We find that the assumption is well supported by our simulation result that pdf in the dissipation scales, such as $1 / 512 L$, and the resolution scale, is very close to that extrapolated from larger, inertial range scales, such as $1 / 128 L$ and $1 / 256 L$.

Reynolds numbers in interstellar turbulence are much larger than can be resolved by current numerical simulations. Because the intermittency theory can only be verified by simulations with limited resolution, the extrapolation of the theory to a realistic value of $R e$ remains uncertain. Numerical experiments with much larger resolution are required to test if the parameters of the theory, such as the dimension $d$, are independent of Re. However, based on the usual assumption of turbulence theory that the statistics in the inertial range are universal, we expect that a theoretical model validated by our simulation, which resolves at least a small range of inertial scales, can be applied to turbulent flows with higher Re. If so, the statistics of the dissipation rate extracted from the inertial range of our simulation, and the derived dimension $d$ of the log-Poisson model, are universal.

We will apply the log-Poisson model to different interstellar clouds using the appropriate values of $R e$. For each type of clouds, we calculate the dissipation scale, $\eta$, from the Reynolds number, and use the pdf, eq. (9), at $l=\eta$, for the distribution of the turbulent heating rate. 


\section{ENERGY BALANCE}

The turbulent heating rate per unit volume is given by,

$$
\Gamma_{\mathrm{t}}=n \mu m_{H} \epsilon(\mathbf{x}, t)
$$

where $n$ and $m_{H}$ are the number density and the mass of the hydrogen atom, $\mu$ is the mean molecular weight (we adopt $\mu=2.35$ for molecular clouds and $\mu=1.23$ for atomic clouds), and $\epsilon(\mathbf{x}, t)$ is the turbulent dissipation rate per unit mass discussed in $\S 2$. The heating rate is spatially inhomogeneous due to the intermittency of the turbulent dissipation. This gives rise to temperature fluctuations. We will use the distribution of $\epsilon$ given by eq. (9), and calculate the mass-weighted average temperature and the temperature probability distribution in dense molecular clouds and in cold diffuse atomic clouds.

We first consider conventional heating processes in these clouds, and then compare their effect with that of turbulent heating. Cosmic rays have been thought to be the primary heating source for molecular clouds. The heating rate by cosmic rays depends on the ionization rate, which is very uncertain (e.g., Caselli et al. 1998). The measured ionization rate in massive protostellar envelopes, where photoionization is insignificant, is about $2.6 \times 10^{-17} \mathrm{~s}^{-1}$ (Van der Tak and Van Dishoeck 2000). Assuming that the energy input per ionization is $20 \mathrm{ev}$ (Goldsmith 2001), we take the cosmic-ray heating rate per unit volume to be,

$$
\Gamma_{\mathrm{cr}}=0.8 \times 10^{-27} n \text { ergs } \mathrm{cm}^{-3} \mathrm{~s}^{-1}
$$

where the number density $n$ is in units of $\mathrm{cm}^{-3}$.

In the neutral atomic medium, the dominant heating source is the grain photoelectric heating. We use the photoelectric heating rate given in Wolfire et al. (2003),

$$
\Gamma_{\mathrm{pe}}=1.3 \times 10^{-24} n \epsilon_{\mathrm{pe}} G_{0} \operatorname{ergs~} \mathrm{cm}^{-3} \mathrm{~s}^{-1}
$$

where $G_{0}$ is the FUV flux in units of the integrated field of Habing (1968) and $\epsilon_{\mathrm{pe}}$ is the heating efficiency. Following Wolfire et al., we take $G_{0}=1.7$ for the local ISM. For the typical cold neutral cloud temperature of $100 \mathrm{~K}$, density of $50 \mathrm{~cm}^{-3}$, and electron fraction of $10^{-4}$ (see Fig. 10 or eq. (C15) in Wolfire et al. 2003), we get $\epsilon_{\mathrm{pe}}=1.4 \times 10^{-2}$ (using eq. (20) in Wolfire et al. 2003), and hence $\Gamma_{\mathrm{pe}}=3.1 \times 10^{-26} n$ ergs $\mathrm{cm}^{-3} \mathrm{~s}^{-1}$.

For molecular clouds, we take the cooling rate per unit volume, $\Lambda_{\mathrm{g}}$, from Table 2 of Goldsmith (2001), which gives coefficients and indices for power-law fits to the temperature dependence of the cooling rates at densities from $10^{2}$ to $10^{7} \mathrm{~cm}^{-3}$. We use a linear interpolation to get coefficients and indices for densities not given in the table. The table is only for velocity gradient of $1 \mathrm{~km} \mathrm{~s}^{-1} \mathrm{pc}^{-1}$. According to eqs (2), (3) and (4) in Goldsmith and Langer (1976), the cooling rate per particle depends on the velocity gradient, $d v / d r$, and on the density only through their product, $n /(d v / d r)$. Therefore, Table 2 of Goldsmith (2001) can be converted to one for the cooling rate per particle as a function of $n(d v / d r)$. We then obtain the cooling rate per particle for a given density and velocity gradient in the range of $n /(d v / d r)$ corresponding to the density range in the table.

For cold neutral HI clouds, the dominant cooling process is the line cooling by the CII $158 \mu \mathrm{m}$ fine structure transition. Considering the low electron fraction $\left(10^{-4}\right)$ in these clouds, we neglect the contribution to the CII cooling from collisional excitation by electrons. In $\S 4.3$, we compute the fraction of gas in cold diffuse clouds with high enough temperature ( $\gtrsim 1000 \mathrm{~K}$ ) to activate the chemical reactions producing $\mathrm{CH}^{+}$. We therefore include cooling by the OI $63 \mu \mathrm{m}$ line, which becomes more important than CII cooling at $T>1000 \mathrm{~K}$. We adopt the cooling rates for CII and OI lines from Wolfire et al (2003). Note that this cooling rate is smaller than that given by Dalgarno and McCray (1972) by a factor of a few. This is because Wolfire et al. (2003) adopted $\mathrm{C}$ and O abundances derived from observations of UV absorption lines by the interstellar gas over many lines of sight studied by HST/FUSE, which are smaller than the solar abundances used in Dalgarno and McCray (1972) by a factor of 2-3.

In our calculations, we will consider energy exchange between gas and dust grains and solve the coupled energy balance equations for gas and dust grains simultaneously. For the energy transfer rate from gas to dust grains, $\Lambda_{\mathrm{gd}}$, we take the formula,

$$
\Lambda_{\mathrm{gd}}=0.7 \times 10^{-33} n^{2} T^{1 / 2}\left(T-T_{\mathrm{d}}\right) \operatorname{ergs~} \mathrm{cm}^{-3} \mathrm{~s}^{-1}
$$

from Goldsmith (2001), where $T$ and $T_{\mathrm{d}}$ are, respectively, the gas and the grain temperatures in units of $\mathrm{K}$ (see also Black 1987, where the rate is larger by a factor of a few). Eq. (13) assumes a dust to gas ratio of 0.01 , grain size of $1.7 \times 10^{-5} \mathrm{~cm}$, grain density of $2 \mathrm{~g} \mathrm{~cm}^{-3}$, and an accommodation coefficient of 0.3 for grains and $\mathrm{H}_{2}$. If $T>T_{d}$, the gas heats the dust and $\Lambda_{\mathrm{gd}}$ is a cooling term for the gas; vice versa, if $T<T_{\mathrm{d}}, \Lambda_{\mathrm{gd}}$ is a heating term for the gas.

Dust grains in molecular clouds are heated by the diffuse UV-visible-IR interstellar radiation. Following Goldsmith (2001), we adopt a flux of $5.3 \times 10^{-3}$ ergs cm $-2 \mathrm{~s}^{-1}$ for this radiation field. Using the above values for the dust to gas ratio and the grain cross section (calculated from the grain size and density), the dust heating rate by the diffuse interstellar radiation is,

$$
\Gamma_{\mathrm{d}}=3.9 \times 10^{-24} n \chi \operatorname{ergs~cm} \mathrm{cm}^{-3} \mathrm{~s}^{-1}
$$

To account for the attenuation of the radiation field due to dust extinction, we have reduced the heating rate by a factor of $\chi$. We take $\chi$ to be $\sim 10^{-1}-10^{-2}$, corresponding to a visual distinction of 2-5 mag., typical of a molecular cloud of size $1 \mathrm{pc}$. The value of $\chi$ affects only the dust temperature in such a cloud (§4.1). For inner regions of high-mass star-forming cores, the radiation from newly formed stars is the primary heating source of dust grains (see $\S 4.2$ ). The heating rate from the stellar radiation gives a high dust temperature. In that case, collisions of gas molecules with dust grains could provide a significant energy source for the molecular gas through $\Lambda_{\mathrm{gd}}$. For dust heating in atomic clouds, we use eq. (14) with $\chi=1$.

The dust cooling rate is calculated by integrating over the wavelength, $\lambda$, the absorption efficiency, $Q_{\lambda}$, times the Planck function (Hollenbach and McKee 1979). For $\lambda \lesssim 100 \mu \mathrm{m}$ (which corresponds to $T_{\mathrm{d}} \lesssim 140 \mathrm{~K}$ ), the absorption efficiency decays as $\lambda^{-2}$ (Draine and Lee 1984; Ossenkolf and Henning 1994). Using $Q_{\lambda} \simeq 4.5 \times 10^{-5}$ at the reference wavelength of $\lambda=790 \mu \mathrm{m}$ (Goldsmith 2001; Ossenkolf and Henning 1994), a numerical evaluation of 
the integral gives,

$$
\Lambda_{\mathrm{d}}=4 \times 10^{-31} n T_{\mathrm{d}}^{6} \operatorname{ergs~cm} \mathrm{cm}^{-3} \mathrm{~s}^{-1}
$$

(Note that this result of our numerical calculation is 60 times larger than that given in Goldsmith (2001)). In our calculations below, the dust temperature is smaller than $140 \mathrm{~K}$, eq. (15) is thus valid for the temperature range of interest here.

Putting together heating and cooling for gas and dust grains and their energy exchange, the energy balance equations for gas and dust grains are, respectively,

$$
\Gamma_{\mathrm{t}}+\Gamma_{\mathrm{cr}}+\Gamma_{\mathrm{pe}}-\Lambda_{\mathrm{g}}-\Lambda_{\mathrm{gd}}=0
$$

and

$$
\Gamma_{\mathrm{d}}+\Lambda_{\mathrm{gd}}-\Lambda_{\mathrm{d}}=0
$$

The energy balance assumption is here justified because the cooling timescale of interstellar clouds we consider in the next section $\left(\sim 10^{4} \mathrm{yr}\right)$ is smaller than their dynamical timescale $\left(\sim 10^{6} \mathrm{yr}\right)$. We will solve these two coupled equations numerically.

\section{APPLICATIONS}

We apply the intermittent turbulent heating model to three different interstellar medium environments: Molecular clouds, high-mass star-forming cores, and cold diffuse HI clouds. The spatial variations in the turbulent heating rate, $\Gamma_{\mathrm{t}}$, given by eq. (10), may result in large spatial fluctuations of the gas temperature. Given the distribution function of $\Gamma_{\mathrm{t}}$, we can calculate the temperature probability function by solving eqs. (16) and (17). However, there is a complication in converting the massweighted distribution of the heating rate to the massweighted temperature distribution. The various terms in eqs. (16) and (17) have different density dependence and thus an exact calculation of the mass-weighted temperature distribution requires the joint statistics of density and dissipation rate fluctuations, which is beyond the scope of this study. We will therefore solve the energy balance equations using simply the average gas density. This is equivalent to assuming that the density field and the dissipation field are not correlated, which is supported by their low correlation coefficient of -0.11 and by Figures 2 and 3 . Although the temperature distribution is not mass-weighted in an exact way, the resulting temperature distribution and the average temperature from our calculations are mass-weighted in the sense that they are computed from the mass-weighted distribution of the dissipation rate. In the case of molecular clouds, the mass-weighting of the temperature distribution discussed in $\S 4.1$ is almost exact. The cooling rate in these clouds has an almost linear dependence on density (Goldsmith and Langer 1978) so, except for the negligible coupling term $\Lambda_{\mathrm{gd}}$, the density dependence in all the terms of eq. (16) is the same.

We point out that the nearly independence between the dissipation rate (per unit mass or per particle) and the density does not imply that the temperature and the density are decoupled. The gas temperature is calculated from the balance between heating and cooling. The cooling rate per particle depends on the density and thus the resulting temperature is correlated with the density through the cooling rate. In this sense, the correlation is similar to the case with a uniform heating rate (as usually assumed for traditional heating sources, e.g., the cosmicray heating): denser regions tend to have a lower temperature than lower density ones. However, the intermittent fluctuations in the turbulent heating rate may also give rise to significant scatter around this general trend. This scatter may be probed through high-resolution molecular emission line maps. In such maps, the observed scatter around the predicted correlation between the projected temperature and density (i.e., averaged along the line of sight) would be smaller than that in 3D space, as can be seen by comparing Fig. 1 with Fig. 2 .

\subsection{Molecular Clouds}

We assume the following Larson's relations for the 1D velocity dispersion and the number density in molecular clouds,

$$
\sigma_{\mathrm{v}}=1(L / 1 \mathrm{pc})^{0.4} \mathrm{~km} \mathrm{~s}^{-1}
$$

and

$$
n=2000(L / 1 \mathrm{pc})^{-1} \mathrm{~cm}^{-3}
$$

where $L$ is the size of the cloud. The average dissipation rate per unit mass is $\bar{\epsilon}=1 / 2\left(\sqrt{3} \sigma_{\mathrm{v}}\right)^{3} / L=$ $0.84 \times 10^{-3}(L / 1 \mathrm{pc})^{0.2} \mathrm{ergs} \mathrm{g}^{-1} \mathrm{~s}^{-1}$, where we used the conversion from the $1 \mathrm{D}$ rms velocity, $\sigma_{\mathrm{v}}$, to the $3 \mathrm{D} \mathrm{rms}$ velocity, $U$, i.e., $U=\sqrt{3} \sigma_{v}$. We have assumed that the turbulence is driven at the length scale of the cloud (see Basu and Murali 2001, who find that driving at smaller scale, for example by stellar outflows, would result in a turbulent heating rate that gives a $\mathrm{CO}$ luminosity in excess of the observations). Therefore, the average turbulent heating rate per unit volume, given by eq. (10), is,

$$
\bar{\Gamma}_{\mathrm{t}}=3 \times 10^{-27} n(L / 1 \mathrm{pc})^{0.2} \operatorname{ergs~cm}^{-3} \mathrm{~s}^{-1}
$$

In reality, the turbulence may be driven at a scale much larger than the cloud size, as suggested by various studies of the velocity scaling in molecular clouds (e.g., Ossenkopf and Mac Low 2002; Heyer and Brunt 2004; Padoan et al. 2006). However, from eq. (20), $\Gamma_{\mathrm{t}}$ is almost independent of $L$, and therefore eq. (20) is accurate within a factor of a few. By adopting the cloud size as the driving scale, we make a conservative assumption, which may slightly underestimate the turbulent heating rate and the resulting gas temperature.

Comparing eq. (20) with eq. (11), we see that the average turbulent heating rate in molecular clouds is of the same order of the cosmic ray heating rate, and possibly a few times larger. This average turbulent heating alone could maintain a temperature of $\sim 10 \mathrm{~K}$ in molecular clouds, and thus the cosmic ray heating, whose rate is highly uncertain, is not even needed to explain the observed gas temperatures. Neglecting the spatial fluctuations in the turbulent heating rate, and using the average heating rate from eq. (20) in eq. (16), and the dust heating rate from eq. (14) in eq. (17), we find that the turbulent heating alone gives a temperature of $17 \mathrm{~K}$ for a 1-pc cloud. Similar temperatures are obtained for clouds of size $0.1 \mathrm{pc}$ and $10 \mathrm{pc}$, because the heating rate from eq. (20) depends very weakly on $L$. In the density range of these clouds $\left(200-2 \times 10^{4}\right)$, the coupling between gas and dust is week, so $\Lambda_{\mathrm{gd}}$ is negligible in comparison with the gas and dust heating rates, and can be neglected. 
The dust and the gas temperatures can be obtained simply from their own energy balance (and thus the gas temperature is independent of $\chi$ in eq. (17)), and, from the interstellar UV-visible-IR radiation field, the dust grains achieve a temperature of $7 \mathrm{~K}$ if $\chi=10^{-2}$, or $10 \mathrm{~K}$ if $\chi=10^{-1}$.

A realistic calculation for the gas temperature needs to take into account the intermittent distribution of the turbulent heating rate. Note that the cooling rate in molecular clouds depends on temperature quite sensitively; for example at $n=1000 \mathrm{~cm}^{-3}, \Lambda_{\mathrm{g}} \propto T^{2.4}$ (Goldsmith 2001). Therefore, given the average temperature, the presence of temperature fluctuations makes the overall cooling rate larger. Conversely, given the average heating rate (and thus the overall cooling rate), the presence of heating rate fluctuations (which give rise to temperature fluctuations) leads to an average temperature lower than from uniform heating. The larger the fluctuation amplitude (for example from stronger intermittency in the heating rate), the smaller the average temperature. This effect can be significant because the log-Poisson distribution of the heating rate, eq. (9), is very skewed toward low values (see Figure 4). In other words, the turbulent heating rate is below its average value in a large fraction of the mass. Because the hotter gas cools faster than the cooler gas, the contribution to the average temperature is mainly from the low-dissipation-rate part of the distribution.

To quantify this intermittency effect, we assume the distribution of the turbulent heating rate, $\Gamma_{t}$, follows the log-Poisson distribution, eq. (99), which we evaluate at the dissipation scale, $\eta$. The dissipation scale is $\eta=L R e^{-3 / 4}$, where the Reynolds number, $R e$, is defined as $R e=U L / \nu$. The kinematic viscosity is calculated as $\nu=c /(n \sigma)$ (see $\S 2)$. We adopt a typical value for the cross section, $\sigma \sim 10^{-15} \mathrm{~cm}^{2}$, and use the average temperature to calculate the sound speed, $c$. By numerically solving eq. (16) and eq. (17), we convert the distribution of $\Gamma_{t}$ into the temperature distribution. We assume the dimension of the intermittent dissipative structures to be $d=1.64$. The cumulative probability of the gas temperature is plotted in Figure 6 for a 1-pc cloud (solid line). We find that the average temperature of the distribution is $8.5 \mathrm{~K}$. As expected, this is lower than $17 \mathrm{~K}$, obtained using the average dissipation rate, but still a reasonable mean temperature for molecular clouds, in the absence of cosmic ray heating.

We estimate the effect of the uncertainty in $d$ measured from snapshot-to-snapshot variations, $1.56<d<1.71$, and find that this range of values of $d$ corresponds to a range of 8.0-8.9 $\mathrm{K}$ for the average temperature, i.e., an uncertainty of only $\sim 6 \%$ around the value of $8.5 \mathrm{~K}$, corresponding to $d=1.64$. The effect on the average temperature is so small because the average temperature is $\sim\left\langle\epsilon^{1 / 2-1 / 3}\right\rangle$ (since the cooling rate is $\sim T^{2-3}$ for the density, $10^{3}-10^{4} \mathrm{~cm}^{-3}$, in the molecular clouds of interest here), which is mainly contributed by the part of the pdf between its peak and the average dissipation rate. From the error bars in Fig. 5, one can see there is little snapshot-to-snapshot variation in this part of the pdf. To further illustrate the effect of the degree of intermittency, we also calculated the average temperature using different values of the dimension of the dissipative structures. For example, if $d=1$, as in the case

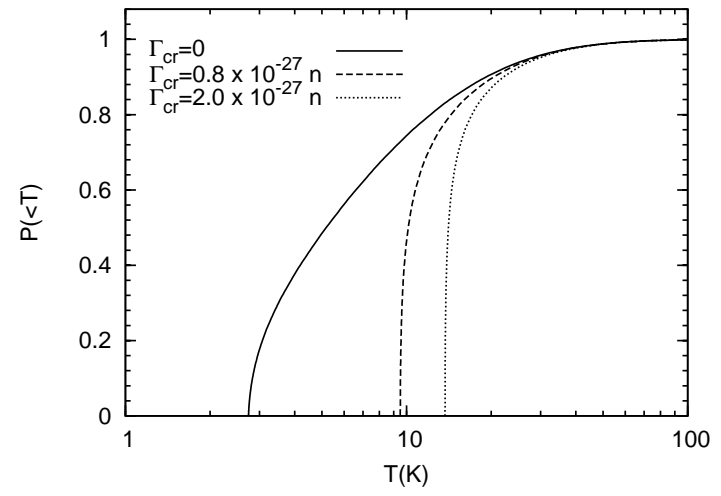

FIG. 6.- Cumulative probability of temperature smaller than $T$ in a molecular cloud of size $1 \mathrm{pc}$. The three curves correspond to the results for different cosmic ray heating rates. Without cosmic ray heating (solid line), the average temperature from turbulent heating is $8.5 \mathrm{~K}$, and $20 \%$ of the gas mass is essentially not heated, due to the intermittency of the turbulent heating rate. When included (dashed and dotted lines), the cosmic ray heating sets a lower limit for the temperature of $9.5 \mathrm{~K}$ and $14 \mathrm{~K}$ (corresponding to the uniform temperature in the absence of turbulent heating), for the two heating rates considered here. In these two cases, the average temperature is $13 \mathrm{~K}$ and $17 \mathrm{~K}$.

of incompressible flows, the dissipation rate distribution would be less intermittent and the average temperature would be $11 \mathrm{~K}$. On the other hand if $d=2$, the average temperature would be lower, about $6 \mathrm{~K}$.

Although the turbulent heating alone gives a reasonable average molecular cloud temperature of $\sim 8.5 \mathrm{~K}$, a significant mass fraction of the clouds receives little heating from turbulent dissipation, because the distribution of the dissipation rate is skewed toward small values. For $d=1.64$, about $20 \%$ of the mass is not significantly heated by turbulence and, in the absence of other heating processes, this mass fraction would be at the same temperature of $2.7 \mathrm{~K}$ as the cosmic microwave background (see Figure 6). The cosmic ray heating would of course dominate in regions with essentially no turbulent heating. In Figure 6] we also show the temperature distribution including both turbulent and cosmic ray heating. To account for the uncertainty in the cosmic ray heating rate, we use two values, $\Gamma_{\mathrm{cr}}=0.8 \times 10^{-27} n \mathrm{ergs} \mathrm{cm}^{-3} \mathrm{~s}^{-1}$ (eq. (11); dashed line) and $\Gamma_{\mathrm{cr}}=2.0 \times 10^{-27} n \mathrm{ergs} \mathrm{cm}^{-3} \mathrm{~s}^{-1}$ (dotted line). The heating rate by cosmic rays sets the lower temperature limit (corresponding to the uniform temperature in the absence of turbulent heating). For $\Gamma=0.8 \times 10^{-27} \mathrm{ergs} \mathrm{cm}^{-3} \mathrm{~s}^{-1}$, this lower limit is $9.5 \mathrm{~K}$. In this case, the average temperature is $13 \mathrm{~K}$ and most gas ( $80 \%$ of the mass) has a temperature lower than the average. The larger cosmic ray heating rate chosen here, $\Gamma_{\mathrm{cr}}=2.0 \times 10^{-27} n$ ergs $\mathrm{cm}^{-3} \mathrm{~s}^{-1}$, gives a lower limit of $14 \mathrm{~K}$ and an average temperature of $17 \mathrm{~K}$. The tail of large turbulent dissipation rate gives a small mass fraction of gas with high temperature. We find that only $1 \%$ of the gas could be hotter than $40 \mathrm{~K}$.

We have shown that the turbulent heating by itself can maintain an average temperature of about $8.5 \mathrm{~K}$ in molecular clouds. Due to the intermittency of this heating process, a finite but small fraction of gas has temperature higher than $40 \mathrm{~K}$, and a significant fraction of the gas is essentially not heated by turbulence, but rather by cosmic rays. In the presence of cosmic rays, turbu- 
lent heating increases the average temperature by a few degrees, from $9.5 \mathrm{~K}$ to $13 \mathrm{~K}$ for the cosmic ray heating rate given by eq. (11).

Similar calculations can be done for dense dark cloud cores. We use the dense cores in the Orion A molecular cloud complex as an example (e.g., Li et al. 2003, 2007 ), and focus on the ones with no apparent internal sources for simplicity ${ }^{1}$. The sizes of these cores are typically 0.05-0.2 pc (e.g., Tatematsu et al. 1993, Li et al. 2003, Ikeda et al. 2007) and the typical average density is $10^{4}-10^{6} \mathrm{~cm}^{-3}$. Observations using tracers such as CS (e.g., Tatematsu et al. 1993), $\mathrm{NH}_{3}$ (e.g., Li et al. 2003), $\mathrm{H}^{13} \mathrm{CO}^{+}$(Ikeda et al. 2007), and other molecular transitions show that the spectral lines have FWHM (full width at half maximum) in the range $0.5-2 \mathrm{~km} \mathrm{~s}^{-1}$, corresponding to $0.22-0.89 \mathrm{~km} \mathrm{~s}^{-1}$ for the $1 \mathrm{D}$ rms velocity, $\sigma_{\mathrm{v}}$. We emphasize that we evaluate the dissipation rate based on the rms velocity, while observational papers usually give the FWHM. We obtain the $1 \mathrm{D}$ rms velocity from the conversion $\sigma_{\mathrm{v}}=\mathrm{FWHM} / 2.355$.

We find that the typical values of $\sigma_{\mathrm{v}}=0.5 \mathrm{~km} \mathrm{~s}^{-1}$ and $L=0.1 \mathrm{pc}$ follow quite closely Larson's relation given in eq. (18), implying that the turbulent heating rate is similar to that in typical regions of molecular clouds discussed above. A complication in evaluating the temperatures in these cores is the possible presence of external UV sources (e.g., from the Trapezium star cluster, see Li et al. 2003). We also need to consider the inner and outer regions of these cores separately. The central region is self-shielded from the diffuse interstellar radiation field or the external UV sources, and thus has a lower dust temperature than the outer regions if there are no internal sources. Furthermore, the gas and dust temperatures are well coupled in the central region, due to the high density $\left(\sim 10^{6} \mathrm{~cm}^{-3}\right)$ (see $\S 4.2$ for a more detailed discussion on the gas-dust coupling). We find that including turbulent heating has little effect to the coupled gas and dust temperature in the central region and the temperature there is essentially determined by the dust heating rate from the diffuse interstellar radiation field and the external UV sources.

At the lower density outer edge, gas and dust are thermally decoupled, and we find that the effect of turbulent heating to the gas temperature is similar to that in the molecular clouds discussed above. As an example, we assume that the dust heating is from the diffuse interstellar radiation field and the rate is given by eq. (14), with $\chi \sim 1$ at the edge. This rate gives a dust temperature of $15 \mathrm{~K}$, close to the average dust temperature of 17 $\mathrm{K}$ found in the observations by Lis et al. (1998). Assuming the gas density is $10^{4} \mathrm{~cm}^{-3}$ at the edge, our model with $d=1.64$ shows that turbulent dissipation alone can heat the gas to an average temperature of $11 \mathrm{~K}$, close to the temperature of $10 \mathrm{~K}$ due to cosmic-ray heating alone. Combining the comic-ray heating with the turbulent heating results in a gas temperature of $14 \mathrm{~K}$, which is in general agreement with the observed temperature (e.g, Li et al. 2003). The effect of turbulent heating here is thus the same as that in molecular clouds stud-

\footnotetext{
${ }^{1}$ In dense cores containing young stellar objects (e.g., those discussed in Johnstone and Bally 2006), one needs to consider heating from the central sources and the calculation would be similar to that for the high-mass star-forming cores to be discussed in $\S 4.2$
}

ied above. Some cases of higher gas temperature have also been found in dense cores in Orion, which can be explained by external UV sources (or internal sources if they are present). UV sources directly heats dust grains and increase the dust temperature. Since in the denser inner regions gas and dust are thermally coupled, the gas temperature there may be increased to the observed higher values by UV sources through energy transfer with dust grains. (The gas temperature at the outer edge with a density $\sim 10^{-4} \mathrm{~cm}^{-3}$ may not increase considerably because the gas and dust temperatures are there decoupled.)

In summary, turbulent heating in central regions of the dense cores in Orion have negligible effects to both gas and dust temperatures, while its role for the outer regions is similar to that in the molecular clouds following Larson's relation. Turbulent heating alone can heat the gas to a temperature of $11 \mathrm{~K}$, and a few degrees higher in combination with cosmic ray heating. The effect of turbulent heating is more prominent in objects with much larger turbulent intensity, such as in the high-mass starforming cores to be discussed in $\S 4.2$, whose average temperature is significantly increased, or in places where the intermittent tail of the heating rate pdf plays an important role for the chemistry, such as in HI clouds, which we study in $\S 4.3$.

\subsection{High-Mass Star-Forming Cores}

We now apply the turbulent heating model to the highmass star-forming cores observed in various CS transitions by Plume et al. (1997). These cores were originally selected by the presence of water masers, suggesting the formation of massive stars, as also shown by their large FIR luminosity (Mueller et al. 2002). The characteristic mean density of these cores is $10^{6} \mathrm{~cm}^{-3}$, the mean size $0.3 \mathrm{pc}$ (average values over the objects listed in Plume et al. (1997) and Shirley et al. (2003)). These cores are very massive, with a typical mass of $\sim 1000 M_{\odot}$ (Plume et al. 1997, Shirley et al. 2003) (much more massive than the dense cores in Orion discussed in $\S 4.1)$. We are interested in these cores because of their extremely large turbulent intensity. The observed FWHM is in the range of $2-12 \mathrm{~km} \mathrm{~s}^{-1}$ (Shirley et al. 2003) with an average of $\sim 6 \mathrm{~km} \mathrm{~s}^{-1}$. This average correspond to a 1D rms velocity of $2.5 \mathrm{~km} \mathrm{~s}^{-1}$. The average turbulent dissipation rate in these dense cores is very large, $\bar{\epsilon}=4.4 \times 10^{-2} \operatorname{ergs~g}^{-1} \mathrm{~s}^{-1}$, approximately 50 times larger than that in a molecular cloud following Larson's relations (see $\S 4.1$ ). However, this heating rate per unit mass, which can be written as $\sim 0.02 L_{\odot} / M_{\odot}$, is much smaller than the average (FIR) bolometric luminosity to mass ratio, $140 L_{\odot} / M_{\odot}$, found by Mueller et al. (2002). Clearly, turbulent heating in these cores could not account for even a tiny fraction of the FIR radiation. The FIR emission must be primarily due to the processed UV photons from the central massive stars, which directly heat the dust grains, resulting in a fairly high dust temperature. The primary gas heating source in these cores is thought to be the energy exchange with dust grains by collisions (Evans 1999). We want to investigate if the turbulent heating may provide a significant energy source for the gas in these dense cores and to estimate the effect of the turbulent heating on the gas temperature. 
The gas density and the dust heating rate from the central stellar sources (which can be estimated from the dust temperature) are needed to calculate the gas temperature from eqs. (16) and (17). The gas density, through the collision frequency, plays a crucial role in determining how well gas and dust grains are thermally coupled (see eq. (13) ). The degree of coupling decreases with the distance to the center, $r$, as the density decreases. The density profile in these cores is usually assumed to be a power-law, $n(r) \propto r^{-\alpha}$, where $\alpha$ is in the range 0.5-2.5 (Mueller et al. 2002). In this range of $\alpha$, most of the gas mass is in the outer regions at large $r$; even for the steepest slope, $\alpha=2.5$, about $70 \%$ of the gas mass is outside $r=0.1 \mathrm{pc}$, for a core of size $0.3 \mathrm{pc}$. Since a large fraction of the gas mass is in the outer regions, one may expect that the density there is about the average density, $10^{6} \mathrm{~cm}^{-3}$, given earlier. However, the measured average density of $10^{6} \mathrm{~cm}^{-3}$ is evaluated from radiative transfer modelling of the emission lines of tracer molecules (Shirley et al. 2003), and thus probably reflects the average density along the line of sight, which is dominated by inner regions for a density profile steeper than $r^{-1}$. In that case, the density at the outer regions is much smaller than $10^{6} \mathrm{~cm}^{-3} .^{2}$ For example, if $\alpha=2$, normalizing the line-of-sight average density to $10^{6} \mathrm{~cm}^{-3}$ and assuming that the radius at the inner edge of the core is $100 \mathrm{AU}$, we find that the density at $0.1 \mathrm{pc}$ is $10^{4} \mathrm{~cm}^{-3}$ (while the density at $0.01 \mathrm{pc}$ is close to the measured average density of $10^{6} \mathrm{~cm}^{-3}$ ). To account for the possibility of a flatter density profile, we take $n$ at $0.1 \mathrm{pc}$ to be $10^{4}-10^{5} \mathrm{~cm}^{-3}$. We did similar estimates for the density at inner regions and found that, almost independent of the slope, $\alpha$, in the range $0.5-2.5$, the gas density at $0.01 \mathrm{pc}$ is always close to the measured average density of $10^{6} \mathrm{~cm}^{-3}$.

Our calculations show that $10^{6} \mathrm{~cm}^{-3}$ is a critical density for the gas and dust temperatures to be well coupled. Thus the gas temperature behavior within $0.01 \mathrm{pc}$ is different from that outside $0.01 \mathrm{pc}$. In our calculations, we will refer to two characteristic values of the radius, $r=0.01 \mathrm{pc}$ and $r=0.1 \mathrm{pc}$, representative of the different gas temperature behaviors in the inner and outer regions of these cores.

The dust temperature depends on the heating rate from the central stellar source, which decreases with increasing radius roughly as $r^{-2}$. Using the cooling rate given by eq. (15), we obtain $T_{\mathrm{d}} \propto r^{-1 / 3}$, which is close to the power-law fit $\left(T_{\mathrm{d}} \propto r^{-0.4}\right)$ to the numerical result from the radiative transfer model in Mueller et al. (2002) (the difference in the power-law indices here may suggest that a radiative transfer calculation gives a dust heating rate decreasing faster than $r^{-2}$, or a dust cooling rate increasing with $T_{\mathrm{d}}$ more slowly than $\left.\propto T_{\mathrm{d}}^{6}\right)$. We find that the energy transfer from dust to gas or from gas to dust (which is possible in the presence of turbulent heating of the gas) is negligible in comparison with the dust heating

\footnotetext{
2 Plume et al. (1997) found that the average density, $\sim 10^{6}$ $\mathrm{cm}^{-3}$, derived from their radiative transfer model, gave a core mass larger than the virial mass by more than an order of magnitude. They realized that the mass discrepancy might have originated from the assumption of uniform density in their model. This suggests that the true average density, determined mainly by the outer regions, is smaller than $10^{6} \mathrm{~cm}^{-3}$ by one or two orders of magnitude, supporting our choice of $10^{4}-10^{5} \mathrm{~cm}^{-3}$.
}

rate from the stellar photons, so the dust temperature is completely determined by the central stellar source. For a typical bolometric luminosity of $10^{4} L_{\odot}$, the dust temperature decreases from about $65 \mathrm{~K}$ at $r=0.01 \mathrm{pc}$, to about $25 \mathrm{~K}$ at $r=0.1 \mathrm{pc}$ (see Fig. 9 in Mueller et al. (2002)).

We now calculate the gas temperatures at $0.01 \mathrm{pc}$ and $0.1 \mathrm{pc}$. We first study the inner region using $T_{\mathrm{d}}=65 \mathrm{~K}$, and a density of $10^{6} \mathrm{~cm}^{-3}$. This dust temperature implies a heating rate of $3 \times 10^{-14}$ ergs $\mathrm{cm}^{-3} \mathrm{~s}^{-1}$ by UV photons from the central stars (estimated from the dust cooling rate, eq. (15)). Fixing this dust heating rate, we first calculate the gas temperature assuming the gas is heated only by collisions with dust grains. By solving eqs. (16) and (17), we find a gas temperature of $57 \mathrm{~K}$, suggesting a strong thermal coupling between gas and dust, as mentioned above. The gas heating rate per unit volume by dust grains is $3.7 \times 10^{-20} \mathrm{ergs} \mathrm{cm}^{-3} \mathrm{~s}^{-1}$, which is only $\sim 10^{-5}$ times the heating rate of the dust grains by the stellar radiation field. Thus the dust temperature is not affected by the energy transfer to the gas. We then calculate the gas temperature at $r=0.01 \mathrm{pc}$ assuming the gas is heated both by the dust and by the turbulence. The average turbulent heating rate in these cores is $1.3 \times 10^{-19}$ ergs $\mathrm{cm}^{-3} \mathrm{~s}^{-1}$, which is larger than the rate from collisions with dust grains when the turbulence is neglected. The inclusion of turbulent heating should therefore result in a gas temperature larger than $57 \mathrm{~K}$. Using again the intermittency model with $d=1.64$, we obtain a mean gas temperature of $64 \mathrm{~K}$, very close to the dust temperature of $65 \mathrm{~K}$. In summary, the gas and the dust temperatures in this dense inner region are very well coupled, but the turbulent heating can still increase the gas temperature by a few degrees.

The outer region is interesting because it contains most of the mass of the core and because its density is lower and so gas and dust are less well coupled. We carry out the same calculation as above for the outer region, at $r=0.1 \mathrm{pc}$, assuming a density of $n=10^{4}-10^{5} \mathrm{~cm}^{-3}$ and a dust temperature of $T_{d}=25 \mathrm{~K}$. Neglecting turbulent heating, we obtain a gas temperature of $17 \mathrm{~K}$ and $6 \mathrm{~K}$, for $n=10^{5}$ and $10^{4} \mathrm{~cm}^{-3}$ respectively (including cosmic ray-heating increases the temperature to $18 \mathrm{~K}$ and $10 \mathrm{~K}$ respectively). In this case the gas temperature is considerably smaller than the dust temperature due to the weak coupling (while with $n=10^{6} \mathrm{~cm}^{-3}$ the gas and dust temperatures would be almost equal). As we did for the inner region, we now include the turbulent heating. For illustration, we assume the average dissipation rate is independent of radius. Without energy transfer between dust and gas, we find that the intermittent turbulent heating alone would give a temperature of $36 \mathrm{~K}$ and $35 \mathrm{~K}$, for $n=10^{4}$ and $10^{5} \mathrm{~cm}^{-3}$ respectively. The gas temperature is here nearly independent of the density because at densities above $10^{4} \mathrm{~cm}^{-3}$ the cooling rate per particle is almost constant (see Goldsmith and Langer 1978). Because in this outer region the thermal coupling between dust and gas is weak, we find that including both the energy transfer between dust and gas and the intermittent turbulent heating, the gas temperature is still $35-36 \mathrm{~K}$. In other words, in the outer region of the cores, containing most of the core mass, the gas is thermally decoupled from the dust and its temperature is completely determined by the turbulent heating. Sim- 
ilar to the case of molecular clouds, we find that range of $d$ from 1.56 to 1.71 results in a small range for the average temperature, within 1.5-2 K (i.e., $\sim 5 \%$ ), around the value $35-36 \mathrm{~K}$ obtained for $d=1.64$.

Our result is in general agreement with the observations of high-mass protostellar objects by Leurini et al. (2007). By fitting the observed methanol spectral lines, they derive the physical parameters of a model consisting of an inner core, an extended component, and an outflow. In particular, they find the kinetic temperature is in range of $22-40 \mathrm{~K}$ in the extended component (corresponding to the outer regions in our model) where the FWHM linewidth is $2.8-4.2 \mathrm{~km}^{-1}$ (see their Table 5) over an average size of $0.1 \mathrm{pc}$ (smaller then the value of $0.3 \mathrm{pc}$ we adopted above). With these specific parameters, and the density range of $10^{5}-10^{6} \mathrm{~cm}^{-3}$ (Table 5 in Leurini et al. 2007), our model would predict a temperature range of $28-39 \mathrm{~K}$. Although the velocity dispersion here is lower than adopted in our example, the size is also smaller, resulting in a dissipation rate and a temperature comparable to those in our example above. Future observations with high spatial resolution will provide a better test of our model.

We conclude that, although negligible in comparison with the dust heating rate by the central stellar sources, turbulent heating in high-mass star-forming cores provides an important energy source for the gas. For a characteristic density of $10^{6} \mathrm{~cm}^{-3}$ in the inner regions, the gas and dust temperatures are well coupled. The turbulent heating increases the gas temperature only by a few degrees. This slight temperature increase in the inner regions may not have important observational or dynamical consequences. On the other hand, the turbulent heating has significant effects in the outer regions, where the density is lower and the coupling of dust and gas is weaker than in the inner regions. For a characteristic dust temperature of $20-30 \mathrm{~K}$ and a density of $10^{-4}-10^{-5}$, the turbulent heating increases the gas temperature from less than 10-20 K (lower than the dust temperature) up to about $36 \mathrm{~K}$. This large increase in the gas temperature by a factor of 2-3 due to turbulent heating, in the region containing most of the core mass, may have important effects on the evolution of the cores, on their star formation efficiency, and on their stellar initial mass function. Future observations of these cores may help determine their gas temperature with sufficient accuracy to constrain the relative importance of turbulent heating.

\subsection{Cold Diffuse HI Clouds}

The observed abundance of $\mathrm{CH}^{+}, \mathrm{HCO}^{+}$, and $\mathrm{OH}$ molecules in cold diffuse clouds (Crane et al. 1995, Gredel 1997, Lucas and Liszt 1996, Liszt and Lucas 1996) suggests the existence of hot regions inside these clouds. The activation temperature of reactions producing these molecules is $>1000 \mathrm{~K}$, while the cloud average temperature is $<100 \mathrm{~K}$. Dissipative heating by MHD shocks and vortex tubes have been proposed to explain the existence of hot regions (see, e.g., Pety and Falgarone 2000). These dissipative structures are the most intermittent structures in the log-Poisson model of the turbulent energy dissipation. Since the model gives the distribution of the dissipation rate at all intensity levels, it can be used to calculate the cumulative probability corresponding to the mass fraction of regions with $T>1000 \mathrm{~K}$. Falgarone and Puget (1995) performed a similar calculation. They used the probability distribution of the velocity difference from experiments with incompressible flows, where the strong dissipative structures responsible for the hot regions are vortex tubes. It is not clear whether their result applies to supersonic turbulence in diffuse neutral clouds, where both shocks and vortex tubes contribute to high temperatures. The log-Poisson intermittency model for supersonic turbulence includes both types of dissipation structures. We will compare the result by Falgarone and Puget (1995) with that from the log-Poisson model for incompressible turbulence and for supersonic flows presented in $\S 2$.

For the characteristic density and length scale of cold neutral clouds we adopt $n=50 \mathrm{~cm}^{-3}$ and $L=1 \mathrm{pc}$ (Helies and Troland 2003). The observed $1 \mathrm{D}$ rms turbulent velocity, $\sigma_{\mathrm{v}}$, at the scale of $1 \mathrm{pc}$, is between 1 and $3 \mathrm{~km} \mathrm{~s}^{-1}$ (Hennebelle et al. 2007). The average turbulent heating rate is $1.5 \times 10^{-27}\left(\sigma_{\mathrm{v}} / 1 \mathrm{~km} \mathrm{~s}^{-1}\right)^{3} \operatorname{ergs~s}^{-1}$ per $\mathrm{H}$ atom. We will treat $\sigma_{\mathrm{v}}$ as a parameter and study the dependence of the mass fraction of hot regions on $\sigma_{\mathrm{v}}$. As discussed in $\S 3$, the photoelectric heating rate in cold neutral clouds is $3.1 \times 10^{-26} \mathrm{ergs} \mathrm{s}^{-1}$ per $\mathrm{H}$ atom. Neglecting turbulent heating, this gives a temperature of 53 $\mathrm{K}$ using the $\mathrm{CII}$ and OI cooling rates from Wolfire et al. (2003). At $n=50 \mathrm{~cm}^{-3}$, the gas-dust coupling is very weak and their energy balance equations can be solved separately.

We now consider the effect of turbulent heating. First we neglect intermittency and use the average dissipation rate, and then we include the effect of intermittency using the $\log$-Poisson model. Assuming $\sigma_{\mathrm{v}}=2 \mathrm{~km} \mathrm{~s}^{-1}$ as an example, we find that the temperature increases to $63 \mathrm{~K}$, much smaller than the $1000 \mathrm{~K}$ required for production of $\mathrm{CH}^{+}$. Temperatures much higher than the average can only be obtained if intermittency is included. In the temperature range from $100 \mathrm{~K}$ to $8000 \mathrm{~K}$, the cooling rate as a function of temperature is very flat, meaning that hotter gas is more difficult to cool. This suggests that temperature fluctuations in this range of values reduce the overall cooling efficiency. Therefore, the net effect of fluctuations in the dissipation rate is that of producing a higher mean temperature than in the case of a uniform dissipation rate (contrary to the case of molecular clouds, where the cooling rate depends on temperature very sensitively). For example, if the dimension of the most intense dissipative structures is $d=1.64$, the average temperature derived for $\sigma_{\mathrm{v}}=2 \mathrm{~km} \mathrm{~s}^{-1}$ is $100 \mathrm{~K}$ (versus $63 \mathrm{~K}$ in the case of a uniform dissipation rate). Furthermore, the flat cooling curve helps the generation of hot regions due to the fluctuations in the dissipation rate, so the extended high-rate tail of the dissipation rate distribution translates into a fairly extended high temperature tail in the temperature distribution.

We calculated the gas temperature probability distribution using $d=1.64$ for the most dissipative structures in the intermittency model. Figure [7 shows the cumulative probability of temperatures lower than $T$ for different rms velocities. From the cumulative probability curve, we can read the fraction of gas with temperature larger than $1000 \mathrm{~K}$. For $\sigma_{\mathrm{v}}=1,2$, and $3 \mathrm{~km} \mathrm{~s}^{-1}$, the probabilities of finding $T>1000 \mathrm{~K}$ are $3.6 \times 10^{-5}$, 


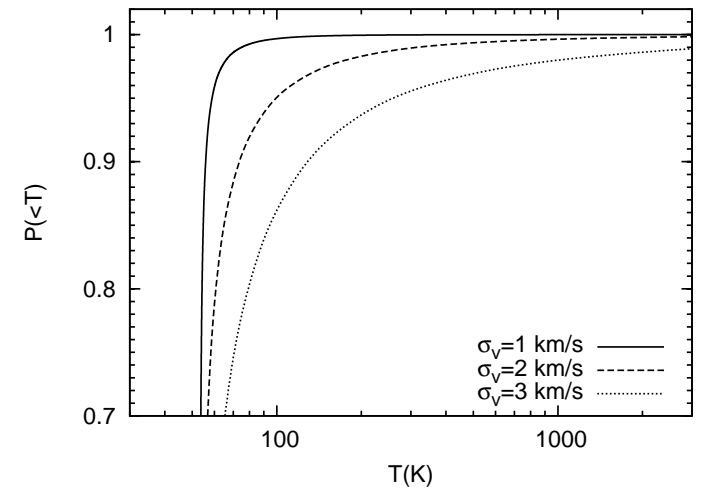

FIG. 7.- Cumulative probability of regions with temperature smaller than $T$ in diffuse HI clouds. The three curves correspond to the results for three different values of the rms turbulent velocity, $\sigma_{\mathrm{v}}=1,2$, and $3 \mathrm{~km} \mathrm{~s}^{-1}$, at the scale of $1 \mathrm{pc}$. The mass fraction of gas with $T>1000 \mathrm{~K}$ for these three cases is $3.6 \times 10^{-5}, 3 \times 10^{-3}$, and 0.02 , respectively. The observed $\mathrm{CH}^{+}$abundance requires that $\sigma_{\mathrm{v}}$ is at least $3 \mathrm{~km} \mathrm{~s}^{-1}$ at $1 \mathrm{pc}$. Most of the gas has a temperature below $100 \mathrm{~K}$.

$3 \times 10^{-3}$ and 0.019 , respectively. Since the hot gas fraction needed to explain the observed abundance of $\mathrm{CH}^{+}$ molecules is a few percent (Gredel et al. 1993), our result shows that cold neutral clouds, where molecules such as $\mathrm{CH}^{+}$are detected, must have a minimum rms turbulent velocity of $3 \mathrm{~km} \mathrm{~s}^{-1}$ at the scale of $1 \mathrm{pc}^{3}$. The uncertainty in $d$ within the range 1.56-1.71 corresponds to a range of the cumulative probability of only $\sim 2$ $3 \%$ around the value of 0.019 found for $d=1.64$ and $\sigma_{\mathrm{v}}=3 \mathrm{~km} \mathrm{~s}^{-1}$. Therefore, the range of $d$ does not affect our conclusion for the minimum rms velocity required to produce enough hot regions to explain the abundance of $\mathrm{CH}^{+}$molecules. One may expect the large uncertainty at the far right tail (Fig. 5) may significantly affect the cumulative probability. However, for $\sigma_{\mathrm{v}}=3 \mathrm{~km} \mathrm{~s}^{-1}$, $1000 \mathrm{~K}$ corresponds to a dissipation rate only 10 times larger than the average (see below for a discussion of a wider range of $d$, between 1 and 2 ). Therefore the cumulative probability is not determined by the far tail, but mainly by the part of the pdf where there are no significant snapshot-to- snapshot variations, as can be seen from Fig. 5. Figure 7 also shows that most of the gas has temperature below $100 \mathrm{~K}$, which is the mean temperature in the case of $\sigma_{\mathrm{v}}=2 \mathrm{~km} \mathrm{~s}^{-1}$. This is because the skewness of the dissipation rate distribution favors values below the average.

To study how the degree of intermittency affects the mass fraction of hot regions, we consider different values (beyond the range 1.56-1.71) of the dimension of the most intense dissipative structures. We choose two special vales, $d=1$ and $d=2$, corresponding to vortex tubes and shocks. The dissipation rate is more intermittent for larger values of $d$. We find that, if $\sigma_{\mathrm{v}}=1 \mathrm{~km} \mathrm{~s}^{-1}$, the

3 We note that Sheffer et al. (2008) find a similar minimum velocity dispersion from a different model proposed by Federman et al. (1996) to explain the observed $\mathrm{CH}^{+}$abundance and its decoupling from the $\mathrm{OH}$ abundance. In their model, reactions between neutral and ionic molecules, e.g., the production of $\mathrm{CH}^{+}$from $\mathrm{C}^{+}$ and $\mathrm{H}_{2}$, are accelerated by the neutral-ion velocity, which is assumed to be the amplitude of MHD waves and is accounted for by using a non-thermal exponent for the effective temperature. We will investigate the intermittent distribution of the neutral-ion velocity in MHD turbulence and its effect on chemistry in HI clouds in a future work. cumulative probability for $T>1000 \mathrm{~K}$ depends on $d$ very sensitively. It is $2 \times 10^{-6}$ and $2 \times 10^{-4}$, respectively, for $d=1$ and $d=2$. However, for $\sigma_{\mathrm{v}}=3 \mathrm{~km} \mathrm{~s}^{-1}$, the degree of intermittency does not affect the probability by much; the cumulative probability is 0.015 and 0.021 for $d=1$ and $d=2$, close to the value of 0.019 that we previously found for $d=1.64$. This is because for $\sigma_{\mathrm{v}}=1 \mathrm{~km} \mathrm{~s}^{-1}$ the average dissipation rate is low, and a temperature of $T=1000 \mathrm{~K}$ requires the far tail of the dissipation rate distribution, which strongly depends on the degree of intermittency. For $\sigma_{\mathrm{v}}=3 \mathrm{~km} \mathrm{~s}^{-1}$, instead, $T=1000 \mathrm{~K}$ corresponds to a dissipation rate only approximately 10 times larger than the average. In the range of values about an order of magnitude around the average, there is no considerable difference in the dissipation rate distribution for different dimensions of the dissipative structures.

Falgarone and Puget (1995) adopted an rms velocity of $3 \mathrm{~km} \mathrm{~s}^{-1}$ at $1 \mathrm{pc}$, and used experimental results for the probability distribution of the dissipation rate of incompressible turbulence (converted from the velocity difference distribution). Their model corresponds to our model with $d=1$. They obtained a mass fraction of a few percent (see their Figure 4) for gas with $T>1000 \mathrm{~K}$, consistent with our result using the same parameters. Therefore, Puget and Falgarone's model, physically valid only if the turbulence in cold atomic clouds were subsonic, gives by chance a result similar to that of our model for supersonic turbulence.

In summary, we have shown that the intermittent turbulent heating can generate enough hot regions to produce the observed $\mathrm{CH}^{+}$abundance in cold neutral clouds if the turbulent rms velocity, $\sigma_{\mathrm{v}}$, is large enough. The required minimum value for $\sigma_{\mathrm{v}}$ is approximately $3 \mathrm{~km} \mathrm{~s}^{-1}$ at a length scale of $1 \mathrm{pc}$.

\section{DISCUSSION AND CONCLUSIONS}

We have studied the energy dissipation and the heating in supersonic turbulence. The turbulent dissipation is characterized by strongly intermittent fluctuations. A significant fraction of the kinetic energy is viscously dissipated in the finest, most intermittent structures, giving rise to a broad tail in the probability distribution function of the dissipation rate. To study the turbulent heating in interstellar clouds, a theoretical model is needed for the probability distribution of the dissipation rate at the dissipation scale, $\eta$. We have generalized the log-Poisson model, originally proposed for incompressible turbulence by She and Leveque (1994), to supersonic turbulence. Because the dissipation scale, $\eta$, cannot be resolved by current numerical simulations, we have used results from resolved inertial-range scales in our numerical simulation of supersonic and super-Alfvénic turbulence as a guideline for the sub-grid scales. We have found that the logPoisson model, with a fractal dimension $d=1.64$ for the most intermittent dissipative structures, gives an excellent fit to the mass-weighted probability distribution of the dissipation rate at resolved scales in the simulation. Extrapolating the model to the corresponding Reynolds numbers, we have studied the turbulent heating in molecular clouds, high-mass star-forming cores, and cold diffuse neutral clouds. Here we summarize our results.

1. In typical molecular clouds, the average turbulent 
heating rate exceeds the cosmic ray heating rate by a factor of 3-4. Fluctuations in the heating rate give a lower mean temperature than expected from the average heating rate; temperature fluctuations make cooling more efficient because in molecular clouds the cooling rate increases sensitively with temperature. Taking intermittency into account, the turbulent heating alone gives a mean temperature of approximately $8.5 \mathrm{~K}$, close to the characteristic temperature of molecular clouds. This would suggest that cosmic rays are not even needed to explain the thermal balance in molecular clouds. However, due to the intermittent fluctuations in the turbulent heating rate, a significant mass fraction of the molecular gas is not heated by the turbulence. Cosmic ray heating would dominate in these regions. Assuming a cosmic ray heating rate of $0.8 \times 10^{-27} n$ ergs $\mathrm{cm}^{-3} \mathrm{~s}^{-1}$, the turbulent heating increases the average temperature by a few degrees, from $9.5 \mathrm{~K}$ to $13 \mathrm{~K}$. We also find that turbulent heating plays a similar role in the outer regions of the dark cloud cores in the Orion molecular cloud complex.

2. Turbulent heating provides an important energy source for the molecular gas in high-mass starforming cores. Assuming spherical symmetry, in the inner regions of these cores (within $\sim 0.01 \mathrm{pc}$ from the center), stellar sources heat the dust grains to a relatively high temperature. Due to the high density of these inner regions $\left(\sim 10^{6} \mathrm{~cm}^{-3}\right)$, the gas and dust are thermally coupled, and the gas temperature is close to the dust temperature even in the absence of turbulent heating. The turbulent heating increases the gas temperature only by a few $\mathrm{K}$. On the other hand, in the outer regions ( $\sim 0.1 \mathrm{pc}$ from the center $)$, where most of the core mass resides, the turbulent heating results in a considerable increase in the gas temperature. The low density of these regions $\left(10^{4}-10^{5} \mathrm{~cm}^{-3}\right)$ makes energy exchange between gas and dusts inefficient and, without turbulent heating, the energy transfer from the dust heats the gas only to $10-20 \mathrm{~K}$. Inclusion of turbulent heating increases the temperature to approximately $36 \mathrm{~K}$. Because turbulent heating causes a large temperature increase in most of the core mass, it may have important implications for the dynamical evolution of the cores and for their star-formation process, and it may also be probed by future observations.

3. The intermittent turbulent heating in diffuse HI clouds can give rise to regions much hotter than the average temperature. These warm regions have been used to explain the existence of molecules such as $\mathrm{CH}^{+}$in HI clouds, whose production needs high temperatures. Using the log-Poisson intermittency model for supersonic turbulence, we find that a turbulent rms velocity of $3 \mathrm{~km} \mathrm{~s}^{-1}$ at $1 \mathrm{pc}$ is sufficient to account for the observed abundance of these molecules, which extends the earlier result, based on incompressible turbulence, by Falgarone and Puget (1995).
We point out that thermal conduction is neglected in our calculations. Conduction tends to transport thermal energy to fill in regions not significantly heated by dissipation, and thus may, to some degree, erase the fluctuations in the heating rate. As discussed in $\S 4$, more intermittent fluctuations in the heating rate give a smaller average temperature in molecular clouds. Therefore, if thermal conduction were included, the average temperature in molecular clouds and in the outer regions of high-mass star-forming cores would be even larger, making turbulent heating even more important in these two cases $(\S 4.1$ and $\S 4.2)$. The situation is different in cold diffuse HI clouds $(\S 4.3)$. If the fluctuations in the heating rate are less intermittent there, the average temperature becomes smaller and, more importantly, the tail of the temperature probability distribution, needed for $\mathrm{CH}^{+}$production, would be less extended. We estimate whether and how much thermal conduction would change our results by calculating the conduction length scale, $l_{c} \simeq \sqrt{\kappa t_{c}}$, during a cooling time scale, $t_{c}$, where $\kappa$ is the thermal conduction coefficient (approximately equal to the kinematic viscosity, $\nu$ ). This is the scale over which thermal conduction can homogenize before the heat from turbulent dissipation is radiated away. We find that in molecular clouds and in HI clouds $l_{c}$ is smaller than (but comparable to) the dissipation length scale, $\eta$. Therefore, the heat generated in the most intermittent structures cannot be transported far from these structures by thermal conduction. This justifies our choice of computing the temperature distribution by using the dissipation rate distribution evaluated at $\eta$. In the outer regions of high-mass star-forming cores, $l_{c}$ is about an order of magnitude larger than $\eta$ (the latter is very small because of the very large Reynolds number). Using the distribution of the dissipation rate at $l_{c}$ instead of $\eta$ (assuming thermal conduction homogenizes the temperature over a size of $l_{c}$ ) gives an average temperature of $40 \mathrm{~K}$ in the outer regions of these cores, a little higher than from the distribution of the dissipation rate evaluated at $\eta$. However, the conduction process in the presence of turbulent motions is more complex than described by the above estimate, and a detailed study of its effects is beyond the scope of this paper.

Although so far neglected, the process of turbulent heating may play an important role in the process of star formation. The mean temperature in molecular clouds defines the mean Jeans mass, which may control the peak of the stellar mass distribution. Because we have found that the mean temperature in molecular clouds and in high-mass star-forming cores may be controlled by turbulent heating, the characteristic stellar mass may be affected by turbulent heating as well. For example, the larger gas temperature predicted in high-mass starforming cores may partly offset their large density, resulting in almost the same characteristic stellar mass, with respect to molecular clouds following Larson's relations. Finally, because the process of turbulent heating results in broad gas temperature distributions, it may be crucial in many molecular chemical reactions, besides those responsible for the formation of $\mathrm{CH}^{+}$molecules.

This research was partially supported by a NASA ATP grant NNG056601G, and by an NRAC allocation 
MCA098020S. We utilized computing resources provided by the San Diego Supercomputer Center, by the National
Center for Supercomputing Applications and by NASA High End Computing Program.

\section{REFERENCES}

Anselmet, F., Gagne, Y., Hopfinger, E. J. \& Antonia, R. A. 1984, J. Fluid Mech. 140, 63.

Arons, J. \& Max, C. E. 1975, ApJ, 196, 177L

Basu, S. \& Murali, C. 2001, ApJ, 551, 743

Black, J. H. 1987, in Interstellar processes, eds. Hollenbach, D. J. and Thronson, H. A., p731

Boldyrev, S., Nordlund, A. \& Padoan, P. 2002, Phys. Rev. Lett., 89, 031102

Bottorff, M. \& Ferland, G. 2002, ApJ, 568, 581

Caselli, P., Walmsley, C. M., Terzieva, R., \& Herbst, E. 1998, ApJ, 499, 234

Crane, P., Lambert, D. L., \& Sheffer, Y. 1995, ApJS, 99, 107

Dalgarno, A. \& McCray, R. A. 1972, ARAA 10, 375

Dennis, T. J. \& Chandran, B. D. G. 2005, ApJ, 622, 205

Draine, B. T. \& Lee, H. M. 1984, ApJ, 285, 89

Dubrulle, B. 1994, Phys. Rev. Lett., 73,959

Evans, N. J. 1999, ARAA, 37, 311

Falgarone, E. \& Puget, J.-L. 1995, A\&A, 293, 840

Frisch, U. 1995, Turbulence. (Cambridge University Press)

Goldreich, P. \& Kwan, J. 1974, ApJ, 189, 441

Goldsmith, P. F. 2001, ApJ, 557, 736

Goldsmith, P. F. \& Langer, W. D. 1978, ApJ, 222, 881

Gredel, R. 1997, A\&A, 320, 929

Gredel, R., van Dishoeck, E. F., \& Black, J. H. 1993, A\&A, 269, 477

Habing, H. J. 1968, Bull. Astron. Inst. Netherlands, 19, 421

Heiles, C., \& Troland, T. H. 2003, ApJ, 586, 1067

Hennebelle, P., Audit, E., \& Miville-Deschenes, M.-A. 2007, A\&A, 465, 445

Heyer, M. H., Brunt, C. M. 2004, ApJ, 615, L45

Hollenbach, D. \& McKee, C. F. 1979, ApJS, 41, 555

Kolmogorov, A. N. 1962, J. Fluid Mech. 13, 82

Kritsuk, A. G., Norman, M. L., Padoan, P., \& Wagner, R. 2007, ApJ, 665, 416

Leurini, S., Schilke, P., Wyrowski, F., \& Menten, K. M. 2007, A\&A, 466, 215

Liszt, H. \& Lucas, R. 1996, A\&A, 314, 917

Lucas, R. \& Liszt, H. 1996, A\&A, 307, 237
Mac Low, M.-M., Klessen, R. S., Burkert, A., \& Smith, M. D. 1998, phys. Rev. Lett., 80, 2754

Mac Low, M.-M. 1999, ApJ, 524, 169

Matthaeus, W. H., Zank, G. P., Oughton, S., Mullan, D. J., \& Dmitruk, P. 1999, ApJ, 523, L94

Minter, A. H. \& Balser, D. S. 1997, ApJ, 484, 133

Mueller, K. E., Shirley, Y. L., Evans, N. J. \& Jacobson, H. R. 2002, ApJS, 143, 469

Muller, W-C. \& Biskamp, D. 2000, Phys. Rev. Lett., 84, 475

Oboukhov, A. M. 1962, J. Fluid Mech. 13, 77

Ossenkopf, V. \& Henning, Th. 1994, A\&A, 291, 943

Ossenkopf, V. \& Mac Low, M.-M. 2002, A\&A, 390, 307

Padoan, P. \& Nordlund, A. 1999, ApJ, 526, 279

Padoan, P., Jimenez, R., Nordlund, A., \& Boldyrev, S. 2004, Phys. Rev. Lett. 2004, 92, 191102

Padoan, P., Juvela, M., Kritsuk, A., \& Norman, M. L. 2006, ApJ, 653, L125

Padoan, P., Nordlund, A., Kritsuk, A. G., Norman, M. L., Li, P. S. 2007, IAUS, 237, 283

Pan, L., Wheeler, J. C., \& Scalo, J. 2008, ApJ, accepted (astro-ph/08031689)

Pety, J. \& Falgarone, E. 2000, A\&A, 356, 279

Plume, R., Jaffe, D. T., Evans, N. J., Martin-Pintado, J., \& Gomez-Gonzalez, J. 1997, ApJ, 476, 730

She, Z-S. \& Leveque, E. 1994, Phys. Rev. Lett, 72, 336

She, Z-S. \& Waymire, E. C. 1995, Phys. Rev. Lett, 74, 262

Shirley, Y. L., Evans, N. J., Young, K. E., Knez, C., \& Jaffe, D. T. 2003, ApJS, 149, 375

Stone, J. M., Ostriker, E. C., \& Gammie, C. F. 1998, ApJ, 508, 99 van der Tak, F. F. S. \& van Dishoeck, E. F. 2000, A\&A, 358, 79L Wolfire, M. G., McKee, C. F., Hollenbach, D., \& Tielens, A. G. G. M. 2003, ApJ, 587, 278

Yaglom, A. M. 1966, Dokl. Akad. Nauk SSSR, 166, 49

Yeung, P. K., Pope, S. B., Lamorgese, A. G. \&Donzis, D. A. 2006, Phys. Fluids. 18, 065103 